\title{
Effect of L-homocysteine on endothelial cell-cell junctions following F-actin stabilization through tropomyosin-1 overexpression
}

\author{
MACIEJ GAGAT $^{1}$, DARIUSZ GRZANKA ${ }^{2}$, MAGDALENA IZDEBSKA $^{1}$ and ALINA GRZANKA ${ }^{1}$ \\ Departments of ${ }^{1}$ Histology and Embryology, and ${ }^{2}$ Clinical Pathomorphology, \\ Nicolaus Copernicus University in Torun, Collegium Medicum in Bydgoszcz, 85-092 Bydgoszcz, Poland
}

Received February 5, 2013; Accepted March 28, 2013

DOI: $10.3892 /$ ijmm.2013.1357

\begin{abstract}
Since the identification of actin in non-muscle cells, it has been suggested that the regulation of the mechanical behaviors of the actin cytoskeleton regulates cellular shape changes and the generation of forces during cell migration and division. The maintenance of cell shape and polarity are important in the formation of cell-cell junctions. The aim of the present study was to determine the effect of L-homocysteine thiolactone hydrochloride on EA.hy926 endothelial cells in the context of the maintenance cell-cell junctions through the stabilization of filamentous actin cytoskeleton (F-actin). The actin filaments were stabilized by the overexpression of tropomyosin-1, which has the ability to stabilize actin filaments in muscle and nonmuscle cells. The stabilization of F-actin induced a significant decrease in the percentage of late apoptotic and necrotic cells following treatment with L-homocysteine. Moreover, the migratory potential of the endothelial cells was greater in the cells overexpressing tropomyosin-1 treated with L-homocysteine. Additionally, our results indicated that the stabilization of F-actin in the EA.hy926 cells significantly increased the expression of junctional $\beta$-catenin, as compared to the cells not overexpressing tropomyosin-1. Similarly, the fluorescence intensity of junctional $\alpha$-catenin was also increased in the cells with stabilized F-actin cytoskeleton. However, this increase was only slightly higher than that observed in the EA.hy926 cells not overexpressing tropomyosin-1. Furthermore, the analysis of Zonula occludens (ZO)-1 relative fluorescence demonstrated a statistically significant decrease in the cell-cell junction areas among the cells with stabilized F-actin cytoskeleton in comparison to the cells not overexpressing tropomyosin-1. Our results indicate that the stabilization of F-actin does not affect the migratory potential of cells, and consequently protects the EA.hy926 cells against the L-homocysteine-induced decrease
\end{abstract}

Correspondence to: Professor Alina Grzanka, Department of Histology and Embryology, Nicolaus Copernicus University in Torun, Collegium Medicum in Bydgoszcz, 24 Karłowicza St., 85-092 Bydgoszcz, Poland

E-mail: agrzanka@cm.umk.pl

Key words: cell-cell junctions, filamentous actin cytoskeleton, $\alpha$-catenin, $\beta$-catenin, Zonula occludens- 1 in cell mobility. Moreover, it is suggested that $\alpha$-catenin may participate in the suppression of actin polymerization in the area of cell-cell junctions. It can be hypothesized that the stabilization of F-actin strengthens endothelial adherens and tight junctions by increasing the number of cell-cell junctions due to the amplification of $\beta$-catenin and the ZO-1 fluorescence signal. However, ZO-1 stabilizes the endothelial barrier function through the stabilization of F-actin and F-actin itself stabilizes the localization of ZO-1.

\section{Introduction}

Since the identification of actin in non-muscle cells, it has been suggested that the regulation of the mechanical behaviors of filamentous actin cytoskeleton (F-actin) regulates cellular shape changes and the generation of forces during cell migration and division (1-3). There are different types of F-actin structures that are crucial in many aspects of cell physiology (4). One of these aspects is the maintenance of cell shape and polarity, which are important in the formation of cell-cell junctions. It has been shown that through the cooperation of junctional actin and F-actin thin bundles, the overall morphological changes may lead to the development of a polarized epithelial cell (5).

Atherosclerosis affects the arteries and results in heart disease and myocardial infarction. Atherosclerosis is caused by injury to the artery endothelium caused by mechanical and environmental factors and the resulting inflammatory response in the vessel wall (6). Previous clinical and experimental studies have indicated that mild to moderate increases in the plasma homocysteine concentration are casual risk factors for vascular disease (7-10). The link between homocysteine and cardiovascular disease has not yet been clearly established, and there is no consensus regarding the mechanism of L-homocysteineinduced endothelial dysfunction (11). One of the proposed mechanisms by which homocysteine induces endothelial dysfunction is vascular cell type-specific oxidative stress, by the formation of intracellular reactive oxygen species (12).

Similar to epithelial cells, endothelial cells have specialized junctional regions that are comparable to adherens junctions and tight junctions. However, whereas in the majority of epithelial cells tight junctions are concentrated at the more apical side of the intercellular cleft, in the endothelium they are frequently intermixed with adherens junctions along the cleft (13). The actin cytoskeleton and associated proteins play a 
vital role in cell-cell adhesion (14). Through their cytoplasmic tails, junctional adhesion proteins may bind to cytoskeletal and signaling proteins, which allows the anchoring of the adhesion proteins to F-actin and the transfer of intracellular signals inside the cell (15-18). The association with actin is required not only for the stabilization of the junctions, but also for the dynamic regulation of junction opening and closure. Although the key role of the actin cytoskeleton in the formation and maintenance of adherens junctions has been recognized due to the molecular links between cadherins and actin filaments (19-21), the structural organization and specific role of the actin cytoskeleton at adherens junctions remain unknown, particularly in endothelial cells (14).

Cultured human origin endothelial cells are widely used as a tool for in vitro studies of endothelial injury (22-25). However, primary endothelial cells, such as human umbilical vein endothelial cells (HUVECs) and immortalized cell lines are not devoid of certain disadvantages (26). In the present study, the EA.hy926 cell line, derived by fusion of HUVECs with the human epithelial cell line, A549 (27), was investigated. EA.hy926 cells have been characterized as regards their morphology and the expression of endothelial-specific markers, and have proven to be useful in endothelial-based studies (27-31).

The aim of the present study was to determine the effect of L-homocysteine on EA.hy926 endothelial cells in the context of the maintenance cell-cell junctions through the stabilization of F-actin. The actin filaments were stabilized by the overexpression of tropomyosin-1, which has the ability to stabilize actin filaments in muscle and non-muscle cells (32-36).

\section{Materials and methods}

Cell culture and treatment. The EA.hy926 immortalized human endothelial cells (ATCC, CRL-2922) were cultured in monolayer at $37^{\circ} \mathrm{C}$ in a humidified $\mathrm{CO}_{2}$ incubator $\left(5 \% \mathrm{CO}_{2}\right)$ in DMEM (Gibco/Invitrogen Life Technologies, Carlsbad, CA, USA) with the addition of $10 \%$ fetal bovine serum (FBS; Gibco/ Invitrogen Life Technologies) and $50 \mu \mathrm{g} / \mathrm{ml}$ of gentamycin (Sigma-Aldrich, St. Louis, MO, USA). The cells were grown in 6-well plates (Falcon/BD Biosciences, Bedford, MA, USA) and treated with $12.5,25$ and $50 \mu \mathrm{M}$ L-homocysteine thiolactone hydrochloride for $24 \mathrm{~h}$. The control cells were grown under the same conditions without L-homocysteine treatment.

Plasmid construction. The tropomyosin-1 $(\alpha)$ isoform 1 (NM 001018005) cDNA was synthesized and subcloned de novo by GeneArt into QIAgenes Expression Construct Insect/Mammalia (Qiagen, Hilden, Germany) based on the pQE-TriSystem vector. Confirmed by restriction analysis and DNA sequencing, transfection-ready and expression-ready constructs were obtained from Qiagen. For the negative control, the $\mathrm{pQE}-$ TriSystem vector (Qiagen) was used. The pmaxGFP ${ }^{\mathrm{TM}}$ control vector for the assessment of transfection efficiency was obtained from Lonza (Basel, Switzerland), as a part of the SE Cell Line 4D-Nucleofector ${ }^{\circledR} \mathrm{X}$ kit (Lonza).

Transfection by nucleofection. For the nucleofection of EA.hy926 cells, the cells were grown up to 80-90\% confluency in DMEM (Gibco/Invitrogen Life Technologies) with the addi- tion of $10 \%$ fetal bovine serum (FBS; Gibco/Invitrogen Life Technologies) and $50 \mu \mathrm{g} / \mathrm{ml}$ of gentamycin (Sigma-Aldrich). Following trypsinization, the suspended cells were transfected using the SE Cell Line 4D-Nucleofector ${ }^{\circledR} \mathrm{X}$ kit according to the manufacturer's instructions. Briefly, a total of $1 \times 10^{6}$ cells were resuspended in $100 \mu \mathrm{l}$ of SE Nucleofector solution, together with $2 \mu \mathrm{g}$ of the plasmid DNA: control vector pmaxGFP, pQETriSystem vector or QIAgenes Expression Construct Insect/ Mammalia with cloned cDNA of tropomyosin-1. The mixture was then transferred into a kit-provided cuvette and the cells were electroporated using 4D-Nucleofector device (Lonza) with program DS-120. Transfection efficiency was analyzed on the day of the experiments by GFP fluorescence intensity analysis using a Tali Image-based cytometer (Invitrogen/ Life Technologies) in the cells transfected with the pmaxGFP control vector.

Western blot analysis. Semi-quantitative analysis of the posttranslational expression of tropomyosin-1 was performed by western blot analysis. The EA.hy926 endothelial cells transfected with the pQE-TriSystem vector or QIAgenes Expression Construct Insect/Mammalia with cloned cDNA of tropomyosin-1 were lysed with RIPA buffer (Sigma-Aldrich). Following normalization of the protein concentration using the BCA protein assay kit (Thermo Scientific Pierce, Rockford, IL, USA) and absorbace using a spectrophotometer, equal amounts of protein (15 $\mu \mathrm{g}$ of total protein per lane) were separated by 4-12\% NuPage Bis-Tris gel (Novex/Life Technologies) and transferred onto nitrocellulose membranes using the iBlot dry western blotting system (Invitrogen/Life Technologies). Pre-stained molecular weight markers (Thermo Scientific Pierce) were used to estimate the position of the protein bands. Subsequently, the membranes were processed using the BenchPro 4100 card processing station (Invitrogen/Life Technologies). The membranes were blocked with $5 \%$ non-fat milk in TBS-T for $2 \mathrm{~h}$ and then incubated with the primary rabbit anti-tropomyosin-1 (Sigma-Aldrich; 1:250) and rabbit anti-GADPH (Sigma-Aldrich; 1:5,000) antibodies diluted in TBS-T for $2 \mathrm{~h}$ at room temperature. After washing with TBS-T, the membranes were incubated with the secondary antibodies conjugated with peroxidase (Sigma-Aldrich; 1:80,000) diluted in TBS-T for $1 \mathrm{~h}$ at room temperature. The immunoreactive bands were visualized by enhanced chemiluminescence (ECL) on CL-XPosure Film (Thermo Scientific). After scanning, the densitometry of the bands was quantified using Quantity One Basic software ver3.6.5 (Bio-Rad, Hercules, CA, USA).

Cell death analysis. The analysis of cell death was performed using a Tali Image-based cytometer and a Tali Apoptosis kit (Invitrogen/Life Technologies) according to manufacturer's instructions. Briefly, following trypsinization, the suspended cells were resuspended in Annexin binding buffer at a concentration of $\sim 5 \times 10^{5}$ to $5 \times 10^{6}$. A total of $5 \mu \mathrm{l}$ of Annexin $\mathrm{V}$ Alexa Fluor 488 was then added to each $100 \mu \mathrm{l}$ of sample and mixed, followed by incubation at room temperature in the dark for $20 \mathrm{~min}$. The cells were then centrifuged and resuspended in $100 \mu \mathrm{l}$ of Annexin binding buffer. After the addition of $1 \mu \mathrm{l}$ of propidium iodide to each sample, the cells were incubated at room temperature in the dark for $3 \mathrm{~min}$. A total of $25 \mu \mathrm{l}$ of stained cells were then loaded into a Tali Cellular Analysis 
Slide (Invitrogen/Life Technologies). The data were analyzed using FCS Express Research Edition software ver4.03 (De Novo Software, Los Angeles, CA, USA) on assumption that viable cells are both Annexin V Alexa Fluor 488- and propidium iodide-negative cells; cells that are in early apoptosis are Annexin V Alexa Fluor 488-positive and propidium iodidenegative; cells that are in late apoptosis are both Annexin V Alexa Fluor 488- and propidium iodide-positive; whereas necrotic cells are Annexin V Alexa Fluor 488-negative and propidium iodide-positive.

In vitro scratch wound healing assay. The EA.hy926 cells transfected with the pQE-TriSystem vector or QIAgenes Expression Construct Insect/Mammalia with cloned cDNA of tropomyosin-1 were seeded into 6-well plates (Falcon/ BD Biosciences) and grown to confluency. The cell monolayer was then subjected to a mechanical scratch wound induced using a $200 \mu \mathrm{l}$ sterile pipette tip. Cells were then cultured for an additional period of $24 \mathrm{~h}$ in the presence or absence of $12.5,25$, or $50 \mu \mathrm{M}$ L-homocysteine thiolactone hydrochloride. Cells in the injured area were visualized under phase-contrast optics (x10 objective) using a TE100-U inverted microscope and photographed using a CCD camera DS-5Mc-U1 and NIS-Elements software ver3.30 (all from Nikon, Tokyo, Japan). The wound area was measured at 0, 3, 6, 12 and $24 \mathrm{~h}$ after treatment of the cells with L-homocysteine thiolactone hydrochloride using ImageJ software ver1.45s (NIH Image).

Fluorescent staining. The EA.hy926 cells transfected with the pQE-TriSystem vector or QIAgenes Expression Construct Insect/Mammalia with cloned cDNA of tropomyosin-1 were seeded into 6-well plates (Falcon/BD Biosciences) and grown on glass coverslips. The cells were then cultured for an additional period of $24 \mathrm{~h}$ in the presence or absence of $12.5,25$, or $50 \mu \mathrm{M}$ L-homocysteine thiolactone hydrochloride and fixed with $4 \%$ paraformaldehyde in PBS, $\mathrm{pH} 7.4$ (15 min, room temperature), blocked in $1 \%(\mathrm{w} / \mathrm{v}) \mathrm{BSA} / \mathrm{PBS}$ and then double stained for junctional proteins and $\mathrm{F}$-actin using antibodies and phalloidin conjugates in the following arrangement: (i) mouse anti- $\alpha$-catenin (Invitrogen/Life Technologies), anti-mouse antibody-Alexa Fluor 488 (Invitrogen/Life Technologies), phalloidin-TRITC (Sigma-Aldrich); (ii) rabbit anti- $\beta$-catenin (Sigma-Aldrich), antirabbit antibody-Alexa Fluor 555 (Invitrogen/Life Technologies) and phalloidin-Alexa Fluor 488 (Molecular Probes/Life Technologies); (iii) mouse anti-Zonula occludens (ZO)-1 (Invitrogen/Life Technologies) anti-mouse antibody-Alexa Fluor 488 (Invitrogen/Life Technologies), phalloidin-TRITC (SigmaAldrich). Cell nuclei were stained with DAPI (Sigma-Aldrich). The slides were mounted in Aqua-Poly/Mount (Polysciences, Inc., Warrington, PA, USA) and examined under a C1 laser-scanning confocal microscope (Nikon) with x100 oil immersion objective. The images from triple labeling were simultaneously collected at the brightest signals of junctional protein using Nikon EZ-C1 software ver3.80 (Nikon). All acquisition parameters, including laser power, pixel dwell time and gains, were kept at the same level for all experiments performed on the same junctional protein.

Measuring of fluorescence intensity. The measurement of fluorescence intensity of each junctional protein and F-actin in the EA.hy926 cells overexpressing tropomyosin-1 and in the cells not overexpressing tropomyosin-1 following treatment with 12.5, 25 and $50 \mu \mathrm{M}$ L-homocysteine thiolactone hydrochloride, was performed on confocal images acquired at the brightest signals of junctional protein at the cell edge. The fluorescence intensity measurement of $\alpha$-catenin, $\beta$-catenin and $\mathrm{ZO}-1$ was performed in whole cells and cell-cell interaction areas using Nikon EZ-C1 software ver3.90 (Gold; Nikon). The relative fluorescence values of junctional proteins were calculated by dividing the fluorescence intensity values measured in the cell-cell interaction areas by the median of the fluorescence intensity measured in the cell-cell interaction areas of the control and the cells transfected with the pQE-TriSystem vector or QIAgenes Expression Construct Insect/Mammalia with cloned cDNA of tropomyosin-1.

Statistical analysis. The data in this study are presented as the means \pm SEM. A two-way ANOVA analysis was performed for the wound healing data, and a two-tailed unpaired t-test was utilized to analyze the statistical significance of the differences between the percentage of the wound area in the EA.hy926 cells transfected with the pQE-TriSystem vector or QIAgenes Expression Construct Insect/Mammalia with cloned cDNA of tropomyosin-1. Statistical comparisons between 2 groups of fluorescence intensity or cell death data were performed using a two-tailed Mann-Whitney U test. A p-value $<0.05$ was considered to indicate a statistically significant difference. GraphPad Prism 5.0 (GraphPad Software) was used for statistical analyses.

\section{Results}

Examination of tropomyosin-1 overexpression. The overexpression of tropomyosin-1 in the EA.hy926 cell line was examined by western blot analysis or the measurement of fluorescence intensity using specific antibodies. Western blot analysis showed a 2.16-fold increase in the expression of tropomyosin-1 in the cells transfected with QIAgenes Expression Construct Insect/Mammalia compared to the cells transfected with the empty plasmid (Fig. 1A). Similarly, the analysis of the fluorescence intensity of tropomyosin-1 labeled cells revealed a 2.01-fold increase in tropomyosin-1 fluorescence intensity, compared to that observed in the cells transfected with the empty pQE-TriSystem vector (Fig. 1B). The transfection efficiency was analyzed using a Tali Image-based cytometer in the EA.hy 926 cells transfected with the pmaxGFP control vector. The average transfection efficiency was $88.87 \%$ (Fig. 1C).

Analysis of cell death using Annexin V Alexa Fluor ${ }^{\circledR} 488$ and propidium iodide assay. The analysis of cell death was performed using a Tali Image-based cytometer following Annexin V Alexa Fluor ${ }^{\circledR} 488$ and propidium iodide double staining. In the EA.hy 926 cells transfected with the empty plasmid, no statistically significant differences in the percentage of live and early apoptotic cells were observed following treatment with all L-homocysteine doses, as compared to the control (Fig. 2A and B). As regards the percentage of late apoptotic cells, we observed an increase from 0.86 to $1.22 \%$ $(\mathrm{P}=0.0284)$ and $1.14 \%(\mathrm{P}=0.0295)$ in the cells treated with 12.5 and $50 \mu \mathrm{M}$ L-homocysteine in comparison to the control, 

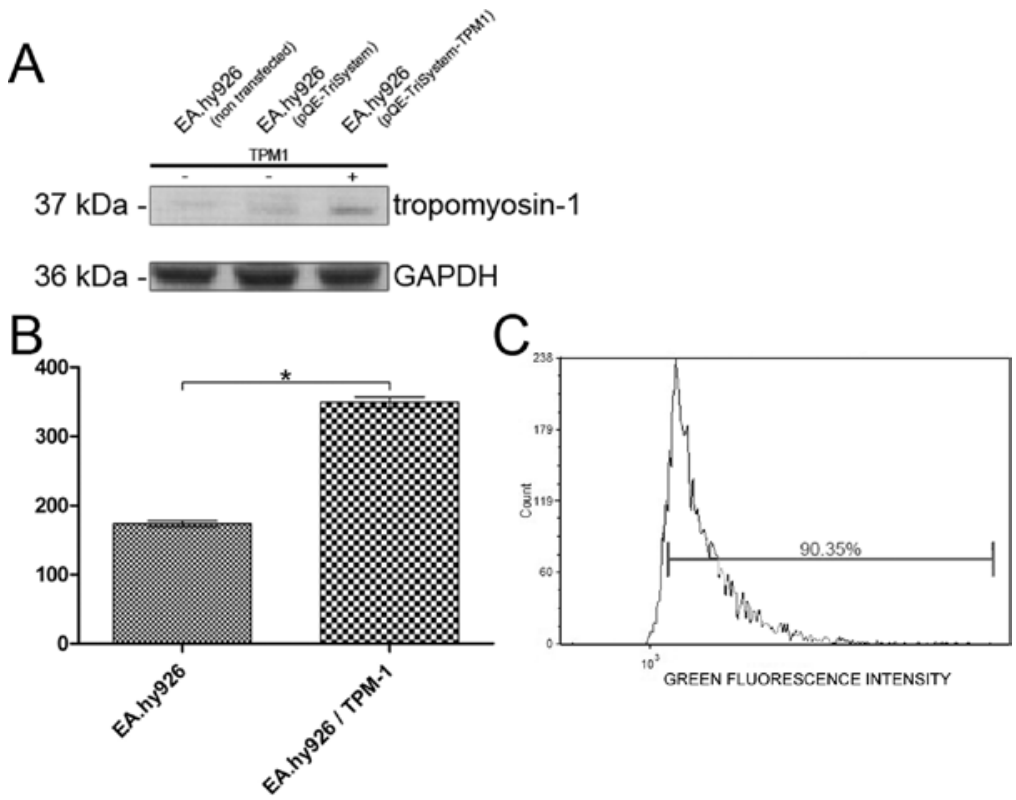

Figure 1. Control of post-transcriptional tropomyosin-1 overexpression and transfection efficiency in the EA.hy926 cell line. EA.hy926 cells were transfected with the QIAgenes Expression Construct Insect/Mammalia encoding tropomyosin-1 ( $\alpha$ ) isoform 1, pQE-TriSystem control vector or pmaxGFP vector. (A) Transfected and non-transfected EA.hy926 cells were lysed and the effect of the empty plasmid or the plasmid with subcloned cDNA of tropomyosin-1 ( $\alpha$ ) isoform 1 on the level of tropomyosin-1 was analyzed by western blot analysis in comparison to the non-transfected cells. (B) EA.hy926 cells transfected with the empty plasmid or the plasmid with subcloned cDNA of tropomyosin-1 $(\alpha)$ isoform 1 were fixed, stained for the tropomyosin-1 protein and examined under a confocal microscope. The analysis of fluorescence intensity of tropomyosin-1 was performed on confocal images. "Statistically significant differences between cells transfected with empty plasmid or plasmid encoding tropomyosin-1 $(\alpha)$ isoform 1; P $<0.05$; Mann-Whitney U test. (C) EA.hy926 cells were transfected with pmaxGFP vector and analyzed for GFP fluorescence using an image-based cytometer.
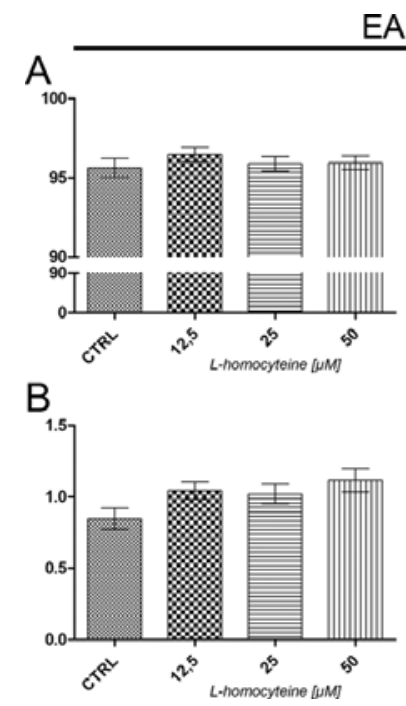

EA.hy926

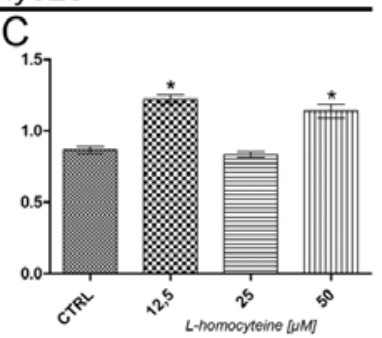

$\mathrm{D}$

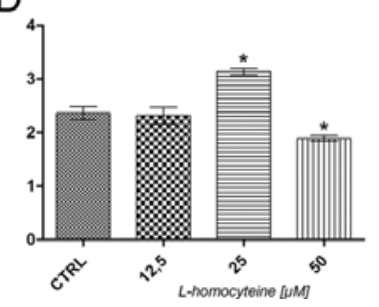

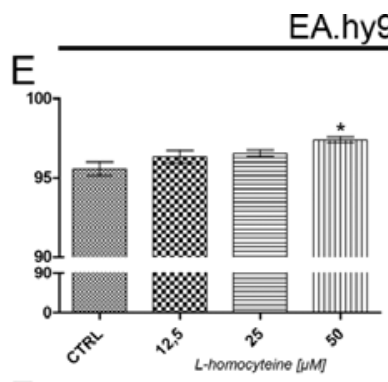

$\mathrm{F}$

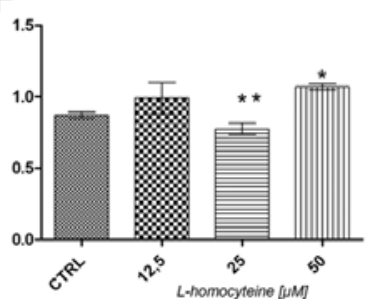

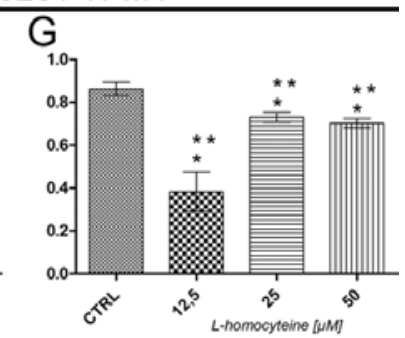

$\mathrm{H}$

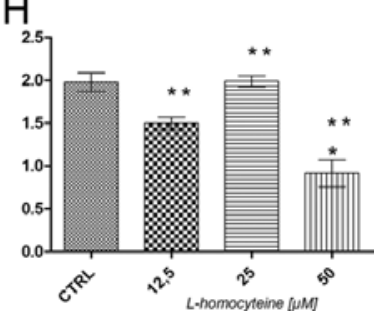

Figure 2. Effect of L-homocysteine thiolactone hydrochloride on the induction of cell death. EA.hy 926 cells transfected with the empty plasmid or the plasmid with subcloned cDNA of tropomyosin-1 $(\alpha)$ isoform 1 were treated with $12.5,25$ and $50 \mu \mathrm{M} \mathrm{L}$-homocysteine thiolactone hydrochloride for $24 \mathrm{~h}$ and doublestained with Annexin V Alexa Fluor 488 and propidium iodide. Analysis was performed using an image-based cytometer. "Statistically significant differences between control cells and cells treated with L-homocysteine; P $<0.05$; Mann-Whitney U test. ${ }^{* *}$ Statistically significant differences between cells transfected with the empty plasmid or plasmid encoding tropomyosin-1 ( $\alpha$ ) isoform 1; P<0.05; Mann-Whitney U test. (A-D) EA.hy926 cells transfected with the empty plasmid. (E and F) EA.hy926 cells transfected with the plasmid encoding tropomyosin-1 ( $\alpha$ ) isoform 1. (A and E) Percentage of live cells. (B and F) Percentage of early apoptotic cells. (C and G) Percentage of late apoptotic cells. (D and H) Percentage of necrotic cells. TPM1, tropomyosin-1.

respectively (Fig. 2C). There was a statistically significant increase in the percentage of necrotic cells (from 2.36 to $3.13 \%$, $\mathrm{P}=0.0284$ ) following treatment with $25 \mu \mathrm{M} \mathrm{L}$-homocysteine and a decrease in the cells treated with the dose of $50 \mu \mathrm{M}$ (to $1.89, \mathrm{P}=0.0396$ ) (Fig. 2D).

In the EA.hy926 cells overexpressing plasmid-encoded tropomyosin-1, a statistically significant increase in the percentage of live (from 95.56 to $97,40 \%, \mathrm{P}=0.0284$ ) and early apoptotic cells (from 0.87 to $1.07 \%, \mathrm{P}=0.0284$ ) was observed following treatment of the cells with $50 \mu \mathrm{M} \mathrm{L}$-homocysteine, as compared to the control (Fig. 2E and F). Moreover, the percentage of late apoptotic cells decreased following treatment of the cells with all L-homocysteine doses in comparison to the control (from 0.86 to $0.38 \%, \mathrm{P}=0,0284 ; 0.73 \%, \mathrm{P}=0.0275$; and 
A

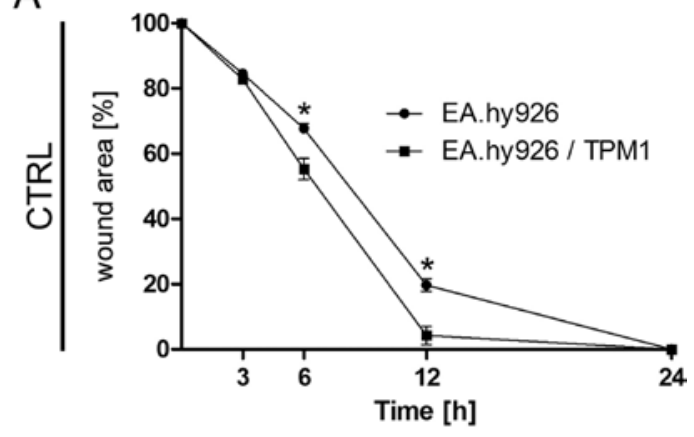

B
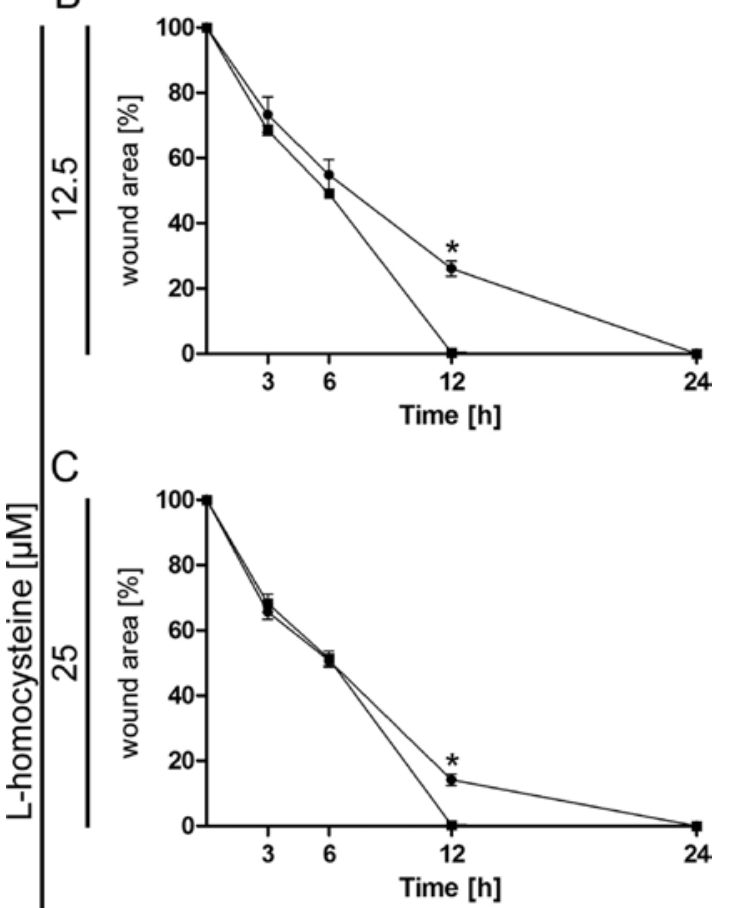

D

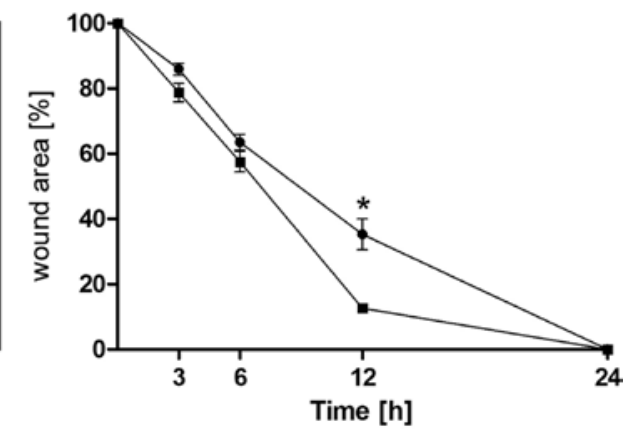

Figure 3. Effect of L-homocysteine thiolactone hydrochloride on cell mobility. EA.hy926 cells transfected with the empty plasmid or the plasmid with subcloned cDNA of tropomyosin-1 $(\alpha)$ isoform 1 were grown to confluency, subjected to a mechanical scratch-wound induction and treated with 12.5, 25 and $50 \mu \mathrm{M}$ L-homocysteine thiolactone hydrochloride for $24 \mathrm{~h}$. The wound area was photographed under phase-contrast optics and measured at 0,3 6, 12 and $24 \mathrm{~h}$ after treatment of the cells with L-homocysteine thiolactone hydrochloride. *Statistically significant differences between cells transfected with empty plasmid or plasmid encoding tropomyosin-1 $(\alpha)$ isoform $1 ; \mathrm{P}<0.05$; unpaired t-test. (A) Control EA.hy926 cells transfected with the empty plasmid or the plasmid encoding tropomyosin-1 ( $\alpha$ ) isoform 1. (B) EA.hy926 cells transfected with the empty plasmid or the plasmid encoding tropomyosin-1 $(\alpha)$ isoform 1 and treated with $12.5 \mu \mathrm{M}$ L-homocysteine thiolactone hydrochloride. (C) EA.hy926 cells transfected with the empty plasmid or the plasmid encoding tropomyosin-1 $(\alpha)$ isoform 1 and treated with $25 \mu \mathrm{M}$ L-homocysteine thiolactone hydrochloride. (D) EA.hy926 cells transfected with the empty plasmid or the plasmid encoding tropomyosin-1 $(\alpha)$ isoform 1 and treated with $50 \mu \mathrm{M}$ L-homocysteine thiolactone hydrochloride. TPM1, tropomyosin-1.
$0.70 \%, \mathrm{P}=0.0256$ for the cells treated with $12.5,25$ and $50 \mu \mathrm{M}$ L-homocysteine, respectively) (Fig. 2G). There was a statistically significant decrease in the percentage of necrotic cells following treatment of the cells with $50 \mu \mathrm{M}$ L-homocysteine (from 1.98 to $0.91 \%, \mathrm{P}=0.0286$ ) (Fig. $2 \mathrm{H}$ ).

After an analysis of cell death, the results demonstrated that due to the overexpression of tropomyosin-1, there was a statistically significant decrease in the percentage of late apoptotic and necrotic cells following L-homocysteine treatment as compared to the cells transfected with the empty plasmid (Fig. 2C, D, G and H). Additionally, there were no statistically significant differences observed between the percentage of live cells (Fig. 2A and E); however, the percentage of early apoptotic cells decreased following treatment of the cells with $25 \mu \mathrm{M}$ L-homocysteine in comparison to the EA.hy926 cells not overexpressing tropomyosin-1, treated with the same dose of L-homocysteine (Fig. 2B and F).

Analysis of cell migration using in vitro scratch wound healing assay. Cell mobility was assessed with a wound healing assay. The results indicated that, while the wound was almost completely repaired after $12 \mathrm{~h}$ in the EA.hy926 cells overexpressing plasmid-encoded tropomyosin-1 treated with all used doses of L-homocysteine, the wound borders were still clearly detectable in the cells transfected with the empty plasmid. After $24 \mathrm{~h}$, wound borders were no longer detectable in the EA.hy926 cells overexpressing tropomyosin-1 and in the cells not overexpressing tropomyosin-1 (Fig. 3A-D). Statistical analysis using an unpaired t-test revealed a significant decrease in the percentage of the wound area in the control EA.hy926 cells overexpressing tropomyosin-1 and in the cells not overexpressing tropomyosin-1 after 6 and $12 \mathrm{~h}$ from the time when the wound was formed (from 67.74 to $55.28 \%, \mathrm{P}=0.0250$ and from 19.71 to $4.26 \%, \mathrm{P}=0.0104$, respectively) (Fig. 3A). Following treatment of the cells with L-homocysteine, cell mobility measured as the percentage of the wound area was also higher in the cells overexpressing tropomyosin-1, as compared to the cells transfected with the empty plasmid after $12 \mathrm{~h}$ from the time when the wound was formed (from 26.09 to $0.33 \%, \mathrm{P}=0.0004$; from 14.20 to $0.40 \%, \mathrm{P}=0.0013$; and from 35,29 to $12.62 \%, \mathrm{P}=0.0096$ for cells treated with $12.5,25$ and $50 \mu \mathrm{M}$ L-homocysteine, respectively) (Fig. 3B-D). Moreover, a comparison of the cells overexpressing tropomyosin-1 and those not overexpressing tropomyosin-1 using two-way ANOVA indicated that time (duration of the experiment) played a crucial role in the control cells and the cells treated with 12.5, 25 and $50 \mu \mathrm{M}$ L-homocysteine.

Fluorescence staining of $\alpha$-catenin and F-actin. The effect of L-homocysteine on the fluorescence staining of $\alpha$-catenin and F-actin in the EA.hy926 cells overexpressing tropomyosin-1 and in those not overexpressing tropomyosin-1 was investigated using a laser scanning confocal microscope. The images were acquired in confocal mode at the focal plane of junctional $\alpha$-catenin. Fluorescence double staining of $\alpha$-catenin and F-actin in the EA.hy926 cells transfected with the empty plasmid revealed a decrease in fluorescence labeling of $\alpha$-catenin following treatment with $12.5,25$ and $50 \mu \mathrm{M}$ L-homocysteine, as compared to the control (Fig. 4A, D, G and J). The weakest labeling intensity of $\alpha$-catenin was observed following treat- 

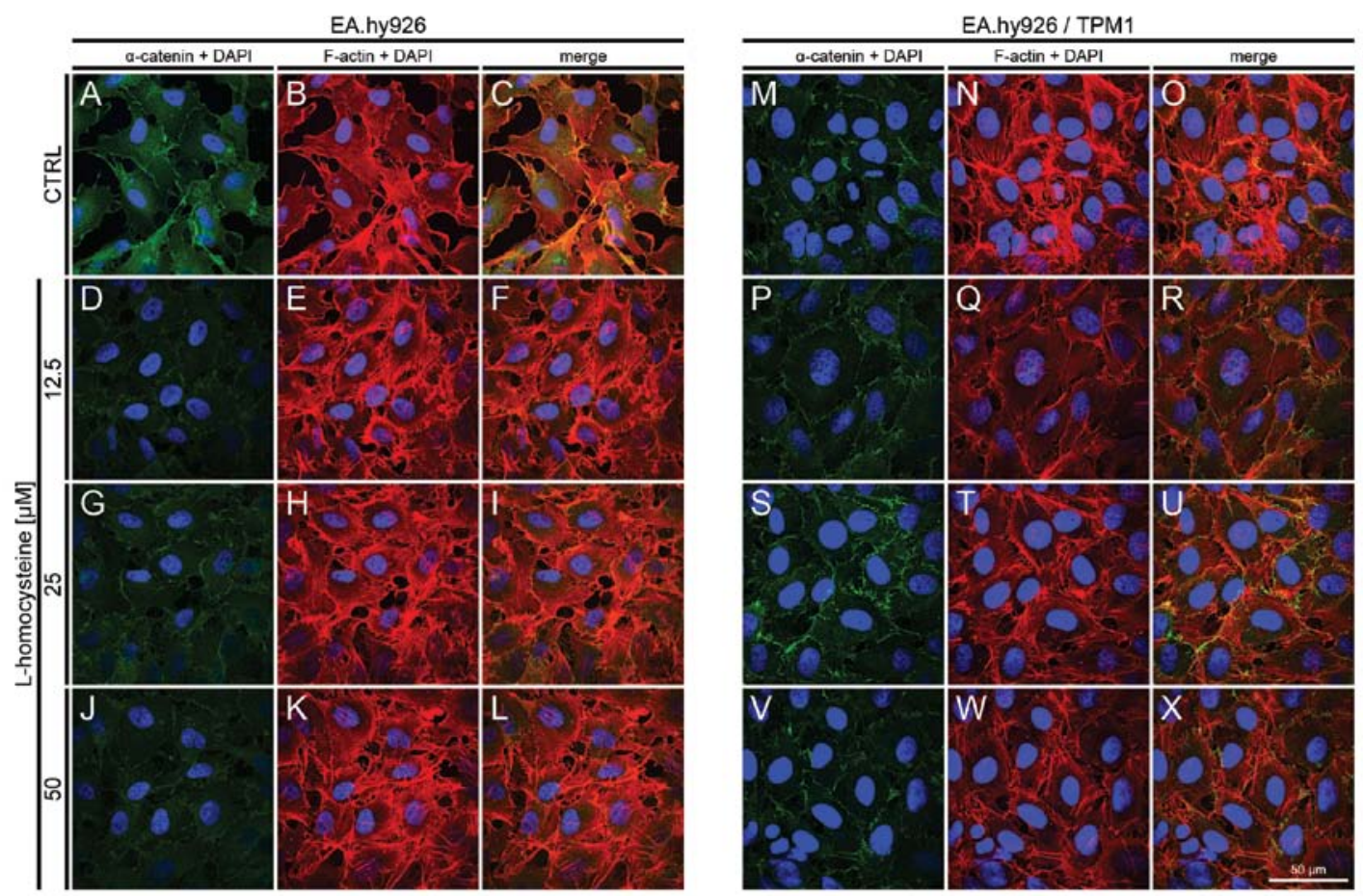

Figure 4. Effect of L-homocysteine thiolactone hydrochloride on fluorescence staining of $\alpha$-catenin and F-actin. EA.hy 926 cells transfected with the empty plasmid or the plasmid with subcloned cDNA of tropomyosin-1 $(\alpha)$ isoform 1 were treated with L-homocysteine thiolactone hydrochloride for $24 \mathrm{~h}$, fixed and double-stained for $\alpha$-catenin and F-actin. Nuclei were counterstained with DAPI. Images were captured at the focal plane of junctional $\alpha$-catenin under a confocal microscope. Bar, $50 \mu \mathrm{m}$. (A-C) Control EA.hy926 cells transfected with the empty plasmid. (D-F) EA.hy926 cells transfected with the empty plasmid and treated with $12.5 \mu \mathrm{M} \mathrm{L}$-homocysteine thiolactone hydrochloride. (G-I) EA.hy926 cells transfected with the empty plasmid and treated with $25 \mu \mathrm{M}$ L-homocysteine thiolactone hydrochloride. (J-L) EA.hy926 cells transfected with the empty plasmid and treated with $50 \mu \mathrm{M}$ L-homocysteine thiolactone hydrochloride. (M-O) Control EA.hy926 cells transfected with the plasmid encoding tropomyosin-1 ( $\alpha$ ) isoform 1. (P-R) EA.hy926 cells transfected with the plasmid encoding tropomyosin-1 $(\alpha)$ isoform 1 and treated with $12.5 \mu \mathrm{M} \mathrm{L}$-homocysteine thiolactone hydrochloride. (S-U) EA.hy926 cells transfected with the plasmid encoding tropomyosin-1 $(\alpha)$ isoform 1 and treated with $25 \mu \mathrm{M} \mathrm{L}$-homocysteine thiolactone hydrochloride. (V-X) EA.hy926 cells transfected with the plasmid encoding tropomyosin-1 $(\alpha)$ isoform 1 and treated with $50 \mu \mathrm{M} \mathrm{L}$-homocysteine thiolactone hydrochloride. (A, D, G and J; M, P, S and V) $\alpha$-catenin + DAPI. (B, E, H and K; N, Q, T and W) F-actin + DAPI. (C, F, I and L; O, R, U and X) Merge. TPM1, tropomyosin-1.

ment of the cells with $12.5 \mu \mathrm{M}$ L-homocysteine (Fig. 4D). By contrast, F-actin labeling revealed a dose-dependent increase in $\mathrm{F}$-actin fluorescence following treatment with L-homocysteine in the form of thick stress fibers (Fig. 4B, E, H and $\mathrm{K}$ ). Moreover, the extracellular spaces were reduced in size following treatment with L-homocysteine in comparison to the control (Fig. 4A-L).

In the EA.hy926 cells with stabilized F-actin cytoskeleton by the overexpression of tropomyosin-1, the labeling of $\alpha$-catenin following exposure of the cells to L-homocysteine was also decreased in comparison to the control and EA.hy 926 cells transfected with the empty plasmid (Fig. 4M, P, S and V). However, $\alpha$-catenin fluorescence at the cell periphery area was increased, as compared to the cells without stabilization of F-actin by tropomyosin-1 (Fig. 4M, P, S and V). Following the stabilization of F-actin, $\alpha$-catenin fluorescence decreased after treatment with L-homocysteine and stress fibers were thinner than those observed in the control cells and in the cells transfected with the empty plasmid (Fig. 4N, Q, T and W). Additionally, the extracellular spaces were significantly reduced following F-actin stabilization (Fig. 4A-X).

The measurement of fluorescence intensity of $\alpha$-catenin and F-actin in the EA.hy926 cells and the cells overexpressing tropomyosin-1 was performed on confocal images acquired at the focal plane of $\alpha$-catenin. The fluorescence intensity of
F-actin and $\alpha$-catenin was measured in whole cells and in cell-cell interaction areas, at the focal plane of $\alpha$-catenin. The analysis of the fluorescence intensity of $\alpha$-catenin measured in whole EA.hy926 cells transfected with the empty plasmid revealed a statistically significant decrease in comparison to the control (from 687.18 to $332.00, \mathrm{P}<0.0001 ; 552.23, \mathrm{P}=0.0148$; and $429.41, \mathrm{P}<0.0001$ for the cells treated with $12.5,25$ and $50 \mu \mathrm{M}$ L-homocysteine, respectively). Additionally, statistically significant differences were obsereved when comparing the different groups (Fig. 5A). The same correlation was observed after the measurement of $\alpha$-catenin fluorescence intensity in the cell-cell interaction areas, although the values were 2.36- to 2.70 -fold higher (Fig. 5B). There was a statistically significant increase in the fluorescence intensity of F-actin measured in whole cells following treatment of the cells with L-homocysteine in comparison to the control (from 1698.91 to 2067.32, $\mathrm{P}=0.0021$; 2063.77, $\mathrm{P}=0.0012$; and 2037.07, $\mathrm{P}=0.0019$ for the cells treated with $12.5,25$ and $50 \mu \mathrm{M}$ L-homocysteine, respectively). The differences between L-homocysteine doses were not statistically significant (Fig. 5C). F-actin fluorescence intensity in the cell-cell interaction areas was statistically significant higher in the cells treated with 25 and $50 \mu \mathrm{M}$ L-homocysteine, as compared to the control (from 2818.09 to 3069.35, $\mathrm{P}=0.0209$ and 3106.83, P=0.0066, respectively). Furthermore, a comparison of the results of F-actin fluorescence intensity measured in 
EA.hy926

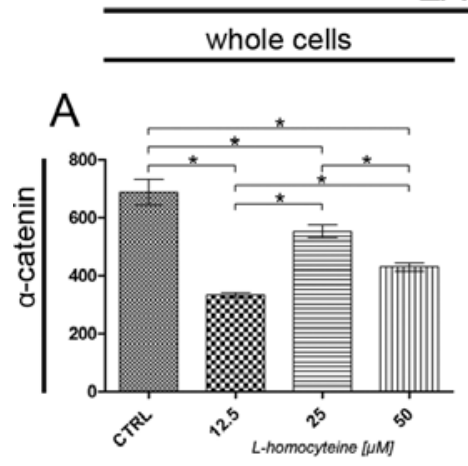

cell-cell interaction areas

B
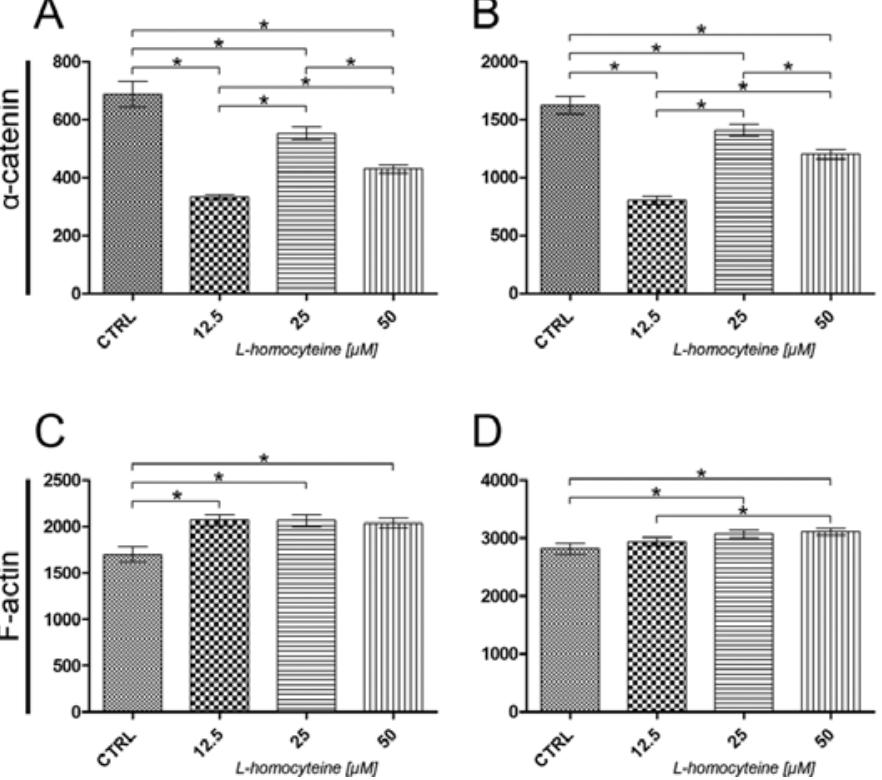

EA.hy926 / TPM1
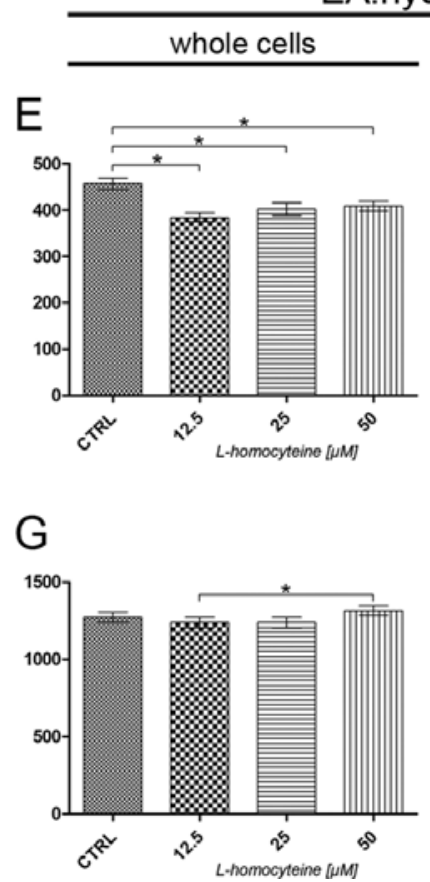

cell-cell interaction areas
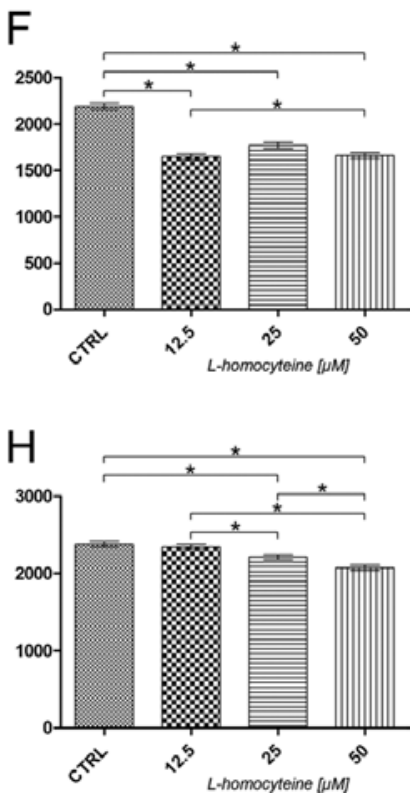

Figure 5. Effect of L-homocysteine thiolactone hydrochloride on the fluorescence intensity of $\alpha$-catenin and F-actin. EA.hy926 cells transfected with the empty plasmid or the plasmid with subcloned cDNA of tropomyosin-1 ( $\alpha$ ) isoform 1 were treated with L-homocysteine thiolactone hydrochloride for 24 h, fixed and double-stained for $\alpha$-catenin and F-actin. Images were captured at the focal plane of junctional $\alpha$-catenin under a confocal microscope. The analysis of fluorescence intensity of $\alpha$-catenin and F-actin was performed on confocal images in whole cells and cell-cell interaction areas. ${ }^{*}$ Statistically significant differences; P<0.05; Mann-Whitney U test. (A and B) Fluorescence intensity of $\alpha$-catenin measured in EA.hy926 cells transfected with empty plasmid. (C and D) Fluorescence intensity of F-actin measured in EA.hy926 cells transfected with the empty plasmid. (E and F) Fluorescence intensity of $\alpha$-catenin measured in EA.hy926 cells transfected with the plasmid encoding tropomyosin-1 $(\alpha)$ isoform 1 . (G and H) Fluorescence intensity of F-actin measured in EA.hy926 cells transfected with the plasmid encoding tropomyosin-1 ( $\alpha$ ) isoform 1. (A and C; E and G) Fluorescence intensity measured in whole cells. (B and D; F and H) Fluorescence intensity measured in cell-cell interaction areas. TPM1, tropomyosin-1; CTRL, control.

the cell-cell interaction areas revealed statistically significant differences between the cells treated with 12.5 and $50 \mu \mathrm{M}$ L-homocysteine (Fig. 5D).

Similar to the results obtained for the cells transfected with the empty plasmid, the measurement of the fluorescence intensity of $\alpha$-catenin in whole EA.hy926 cells overexpressing tropomyosin-1 revealed a statistically significant decrease following treatment with L-homocysteine in comparison to the control (from 456.93 to $383.97, \mathrm{P}<0.0001 ; 401.91$, $\mathrm{P}=0.0021$; and $408.35, \mathrm{P}=0.0016$ for cells treated with 12.5 , 25 and $50 \mu \mathrm{M}$ L-homocysteine, respectively). The differences between the results obtained from the groups of cells treated with L-homocysteine were statistically insignificant. Moreover, the $\alpha$-catenin fluorescence intensity measured in whole cells with stabilized F-actin cytoskeleton was decreased in comparison to that measured in cells transfected with the empty plasmid (Fig. 5E). The same correlation was observed after the measurement of $\alpha$-catenin fluorescence intensity in the cell-cell interaction areas (decrease in fluorescence intensity from 2189.76 to $1646.73, \mathrm{P}<0.0001 ; 1767.05, \mathrm{P}<0.0001$; and $1658.60, \mathrm{P}<0.0001$ for the cells treated with $12.5,25$ and $50 \mu \mathrm{M}$ L-homocysteine, respectively); however, a statistically significant difference was observed between the results obtained for the cells treated with 12.5 and $50 \mu \mathrm{M}$ L-homocysteine. Furthermore, the fluorescence intensity of $\alpha$-catenin measured in the cell-cell interaction areas of EA.hy926 cells with stabilized F-actin cytoskeleton by the overexpression of tropomyosin-1 increased 1.25- to 2.05-fold, as compared to the values measured in the cell-cell interaction area of the cells transfected with the empty plasmid (Fig. 5F).

The measurement of F-actin fluorescence intensity in whole cells overexpressing tropomyosin-1 did not reveal any statistically significant differences, as compared to the control. A comparison of the results of the measurement of F-actin fluorescence intensity following treatment with L-homocysteine revealed statistically significant differences between the cells treated with 12.5 and $50 \mu \mathrm{M}$ L-homocysteine (1237.81 and $1316.38, \mathrm{P}=0.0395$, respectively) (Fig. $5 \mathrm{G}$ ). In contrast to the results obtained for the cells transfected with the empty plasmid, there was a statistically significant decrease in the F-actin fluorescence intensity in the cell-cell interaction areas of the cells overexpressing tropomyosin- 1 in the cells treated with 25 and $50 \mu \mathrm{M}$ L-homocysteine, as compared to the control (from 2379.86 to 2210.09, $\mathrm{P}=0.0006$; and 2070.97, $\mathrm{P}<0.0001$, respectively). Furthermore, a comparison of the results of F-actin fluorescence intensity measured in the cell-cell interaction areas revealed statistically significant differences between the cells treated with all L-homocysteine doses. Additionally, the fluorescence intensity of F-actin measured in the cell-cell interaction areas of the transfected cells decreased by $0.67-$ to 0.84-fold, as compared to the cells transfected with the empty plasmid (Fig. 5H).

Fluorescence staining of $\beta$-catenin and $F$-actin. The effect of L-homocysteine on the fluorescence staining of $\beta$-catenin and F-actin in the EA.hy926 cells overexpressing tropomyosin-1 

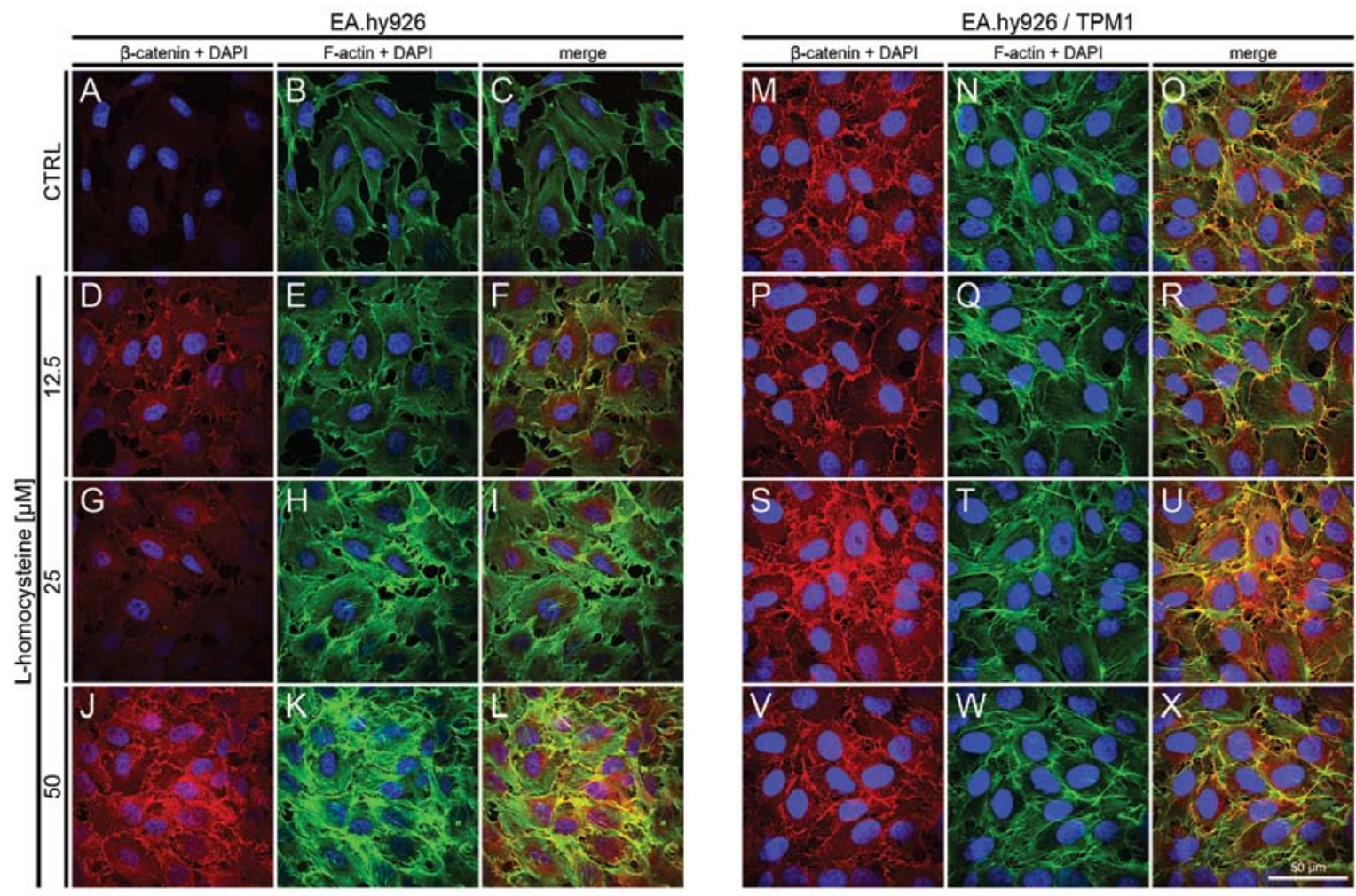

Figure 6. Effect of L-homocysteine thiolactone hydrochloride on fluorescence staining of $\beta$-catenin and F-actin. EA.hy926 cells transfected with the empty plasmid or the plasmid with subcloned cDNA of tropomyosin-1 $(\alpha)$ isoform 1 were treated with L-homocysteine thiolactone hydrochloride for 24 h, fixed and double-stained for the $\beta$-catenin and F-actin. Nuclei were counterstained with DAPI. Images were captured at the focal plane of junctional $\beta$-catenin under a confocal microscope. Bar, $50 \mu \mathrm{m}$. (A-C) Control EA.hy926 cells transfected with the empty plasmid. (D-F) EA.hy926 cells transfected with the empty plasmid and treated with $12.5 \mu \mathrm{M}$ L-homocysteine thiolactone hydrochloride. (G-I) EA.hy 926 cells transfected with the empty plasmid and treated with $25 \mu \mathrm{M}$ L-homocysteine thiolactone hydrochloride. (J-L) EA.hy926 cells transfected with the empty plasmid and treated with $50 \mu \mathrm{M}$ L-homocysteine thiolactone hydrochloride. (M-O) Control EA.hy926 cells transfected with the plasmid encoding tropomyosin-1 ( $\alpha$ ) isoform 1 . (P-R) EA.hy926 cells transfected with the plasmid encoding tropomyosin-1 $(\alpha)$ isoform 1 and treated with $12.5 \mu \mathrm{M}$ L-homocysteine thiolactone hydrochloride. (S-U) EA.hy926 cells transfected with the plasmid encoding tropomyosin-1 $(\alpha)$ isoform 1 and treated with $25 \mu \mathrm{M}$ L-homocysteine thiolactone hydrochloride. (V-X) EA.hy 926 cells transfected with the plasmid encoding tropomyosin-1 $(\alpha)$ isoform 1 and treated with $50 \mu \mathrm{M}$ L-homocysteine thiolactone hydrochloride. (A, D, G and $\mathrm{J}$; M, P, S and V) $\beta$-catenin + DAPI. (B, E, H and K; N, Q, T and W) F-actin + DAPI. (C, F, I and L; O, R, U and X) Merge. TPM1, tropomyosin-1.

and in those not overexpressing tropomyosin-1 was investigated using a laser scanning confocal microscope. The images were acquired in confocal mode at the focal plane of junctional $\beta$-catenin. Fluorescence double staining of $\beta$-catenin and F-actin in the EA.hy926 cells transfected with the empty plasmid revealed an increase in the fluorescence labeling of $\beta$-catenin following treatment with all doses of L-homocysteine in comparison to the control (Fig. 6A, D, G and J). The weakest labeling intensity was observed in the control cells, and the fluorescence at the cell periphery area was only slightly higher than that in the central area of the cells (Fig. 6A). Similar to the results obtained at the focal plane of $\alpha$-catenin, F-actin labeling revealed a dose-dependent increase in fluorescence following treatment with L-homocysteine in the form of thick stress fibers (Fig. 6B, E, H and K), and the extracellular spaces were reduced in size following treatment with L-homocysteine in comparison to the control (Fig. 6A-X).

In the EA.hy926 cells with stabilized F-actin cytoskeleton by the overexpression of tropomyosin-1, the labeling of $\beta$-catenin following exposure of the cells to L-homocysteine was increased in comparison to the control and treated cells, particularly at the doses of 25 and $50 \mu \mathrm{M}$ L-homocysteine. Moreover, the fluorescence of $\beta$-catenin was significantly higher in the control and the cells treated with 12.5 and $25 \mu \mathrm{M}$
L-homocysteine, as compared to the EA.hy926 cells transfected with the empty plasmid. However, $\beta$-catenin fluorescence at the cell periphery area was increased in the cells overexpressing tropomyosin-1 compared to the cells not overexpressing tropomyosin-1 (Fig. 6M, P, S and V). Following the stabilization of F-actin, $\beta$-catenin fluorescence was at a similar level in control and in the cells treated with L-homocysteine. Moreover, stress fibers were thinner than those observed in the control cells and the cells transfected with the empty plasmid (Fig. 6N, Q, $\mathrm{T}$ and $\mathrm{W}$ ). Additionally, the extracellular spaces were significantly reduced following F-actin stabilization, as compared to the cells transfected with the empty plasmid (Fig. 6A-X).

The measurement of fluorescence intensity of $\beta$-catenin and F-actin in the EA.hy926 cells overexpressing tropomyosin-1 and in those not overexpressing tropomyosin-1 was performed on confocal images acquired at the focal plane of $\beta$-catenin. The fluorescence intensity of $\mathrm{F}$-actin and $\beta$-catenin was measured in whole cells and in the cell-cell interaction areas, at the focal plane of $\beta$-catenin. The analysis of the fluorescence intensity of $\beta$-catenin measured in whole EA.hy926 cells transfected with the empty plasmid revealed a statistically significant dosedependent increase in comparison to the control (from 343.79 to $868.79, \mathrm{P}<0.0001 ; 923.96, \mathrm{P}<0.0001$; and $1185.90, \mathrm{P}<0.0001$ for cells treated with $12.5,25$ and $50 \mu \mathrm{M}$ L-homocysteine, 


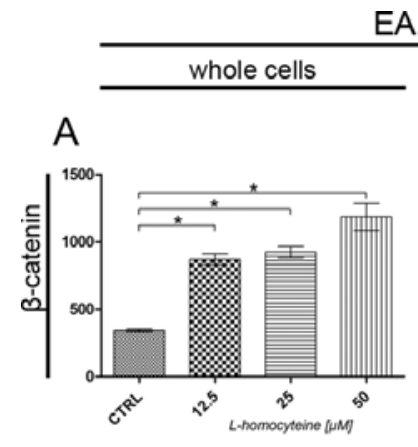

\section{EA.hy926}
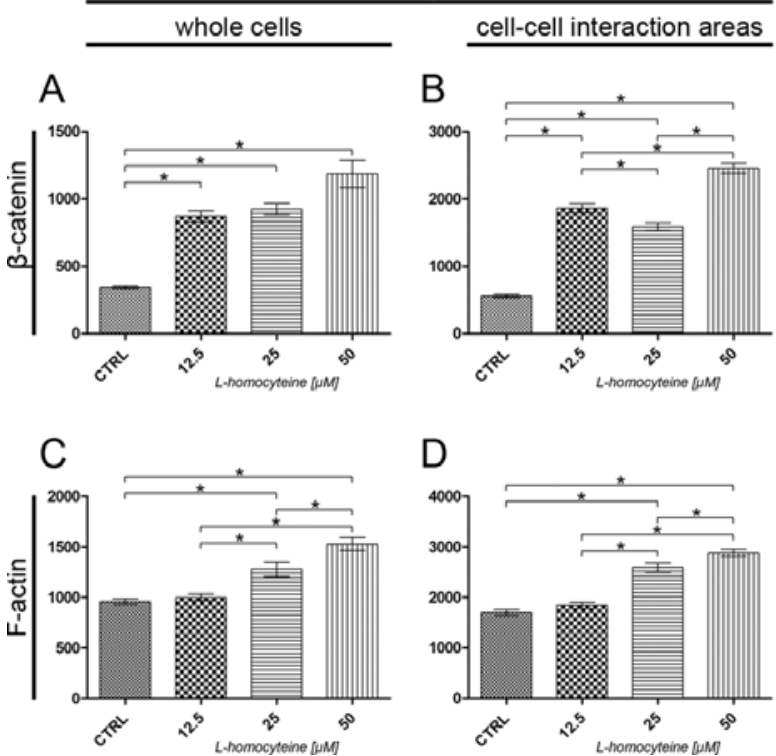

D

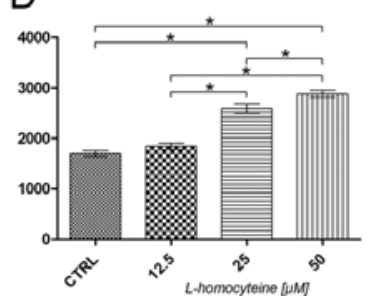

EA.hy926 / TPM1

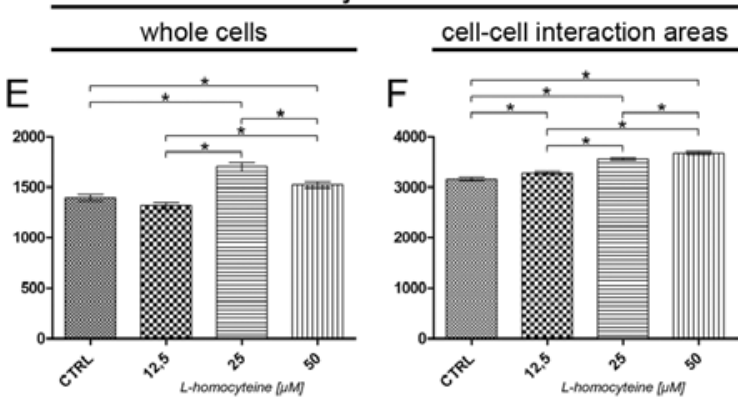

G

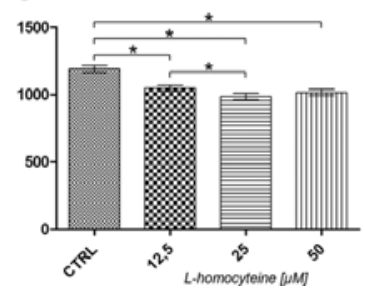

$\mathrm{H}$

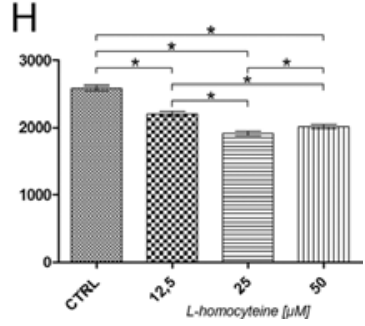

Figure 7. Effect of L-homocysteine thiolactone hydrochloride on the fluorescence intensity of $\beta$-catenin and F-actin. EA.hy926 cells transfected with the empty plasmid or plasmid with subcloned cDNA of tropomyosin-1 $(\alpha)$ isoform 1 were treated with L-homocysteine thiolactone hydrochloride for 24 h, fixed and doublestained for the $\beta$-catenin and F-actin. Images were captured at the focal plane of junctional $\beta$-catenin under a confocal microscope. The analysis of fluorescence intensity of $\beta$-catenin and F-actin was performed on confocal images in whole cells and cell-cell interaction areas. *Statistically significant differences; $\mathrm{P}<0.05$; Mann-Whitney U test. (A-B) Fluorescence intensity of $\beta$-catenin measured in EA.hy 926 cells transfected with the empty plasmid. (C-D) Fluorescence intensity of F-actin measured in EA.hy926 cells transfected with the empty plasmid. (E-F) Fluorescence intensity of $\beta$-catenin measured in EA.hy926 cells transfected with the plasmid encoding tropomyosin-1 $(\alpha)$ isoform 1 . $(\mathrm{G}-\mathrm{H})$ Fluorescence intensity of F-actin measured in EA.hy 926 cells transfected with the plasmid encoding tropomyosin-1 $(\alpha)$ isoform 1. (A and C; E and G) Fluorescence intensity measured in whole cells. (B and D; F and H) Fluorescence intensity measured in cell-cell interaction areas. TPM1, tropomyosin-1; CTRL, control.

respectively). The differences between L-homocysteine doses were statistically insignificant (Fig. 7A). A similar increase in $\beta$-catenin fluorescence in comparison to the control was observed after the measurement of its intensity in the cell-cell interaction areas; however, the values were statistically significant between the L-homocysteine doses used (Fig. 7B). Additionally, the results of the measurement of $\beta$-catenin fluorescence intensity in the cell-cell interaction areas were 1.63- to 2.14-fold higher than the values obtained from whole cells. There was a statistically significant increase in fluorescence intensity of F-actin measured in whole cells following treatment of the cells with 25 and $50 \mu \mathrm{M}$ L-homocysteine in comparison to the control (from 956.89 to $1278.88, \mathrm{P}=0.0002$; and 1528.29, $\mathrm{P}<0.0001$, respectively). Moreover, the differences between L-homocysteine doses were statistically significant (Fig. 7C). A similar correlation was observed in F-actin intensity measured in the cell-cell interaction areas; however, the fluorescence intensity was 1.77 - to 2.02-fold higher than the intensity measured in whole cells (Fig. 7D).

Analysis of the fluorescence intensity of $\beta$-catenin measured in whole EA.hy 926 cells overexpressing tropomyosin-1 revealed a statistically significant increase only in the cells treated with 25 and $50 \mu \mathrm{M}$ L-homocysteine, as compared to the control (from 1293.70 to $1700.32, \mathrm{P}<0.0001$; and 1522,22 $\mathrm{P}=0.0150$, respectively). However, statistically significant differences between L-homocysteine doses were observed. Additionally, the fluorescence intensity values were significantly higher (1.28- to 4.05-fold) than those in the cells transfected with the empty plasmid (Fig. 7E). The fluorescence intensity measured in the cell-cell interaction areas increased in a dose-dependent manner following the stabilization of F-actin cytoskeleton by tropomyosin-1 in the EA.hy926 cells (from 3157.99 to 3283.89 , $\mathrm{P}=0.0026 ; 3557.68, \mathrm{P}<0.0001$; and $3675.25, \mathrm{P}<0.0001$ for the cells treated with 12.5, 25 and $50 \mu \mathrm{M}$ L-homocysteine, respectively). Furthermore, statistically significant differences between the L-homocysteine doses were observed. Similar to the results obtained by measurements in whole cells, the cell-cell interaction areas exhibited significantly higher $\beta$-catenin fluorescence intensity than that observed in the cells without stabilized F-actin (1.50- to 5.64-fold); however, these values were 2.09to 2.41-fold higher than those obtained from measurements in whole cells overexpressing tropomyosin-1 (Fig. 7F).

The measurement of F-actin fluorescence intensity at focal plane of $\beta$-catenin in whole cells overexpressing tropomyosin-1 revealed a statistically significant decrease, as compared to the control (from 1191.57 to 1048.44, $\mathrm{P}<0.0001$; 986.88, $\mathrm{P}<0.0001$; and 1017.10, $\mathrm{P}<0.0001$ for the cells treated with 12.5, 25 and $50 \mu \mathrm{M}$ L-homocysteine, respectively). A comparison of the results of the measurement of F-actin fluorescence intensity following treatment with L-homocysteine revealed statistically significant differences only between the cells treated with 12.5 and $50 \mu \mathrm{M}$ L-homocysteine $(\mathrm{P}=0.0420)$ (Fig. 7G). A similar correlation was observed in F-actin intensity measured in the cell-cell interaction areas; however, the fluorescence intensity was 1.93 - to 2.17 -fold higher than the intensity measured in whole cells. Moreover, statistically significant differences between F-actin fluorescence measured in the cells treated with L-homocysteine were observed (Fig. 7H).

Fluorescence staining of ZO-1 and F-actin. The effect of L-homocysteine on the fluorescence staining of ZO-1 and F-actin in the EA.hy926 cells overexpressing tropomyosin-1 

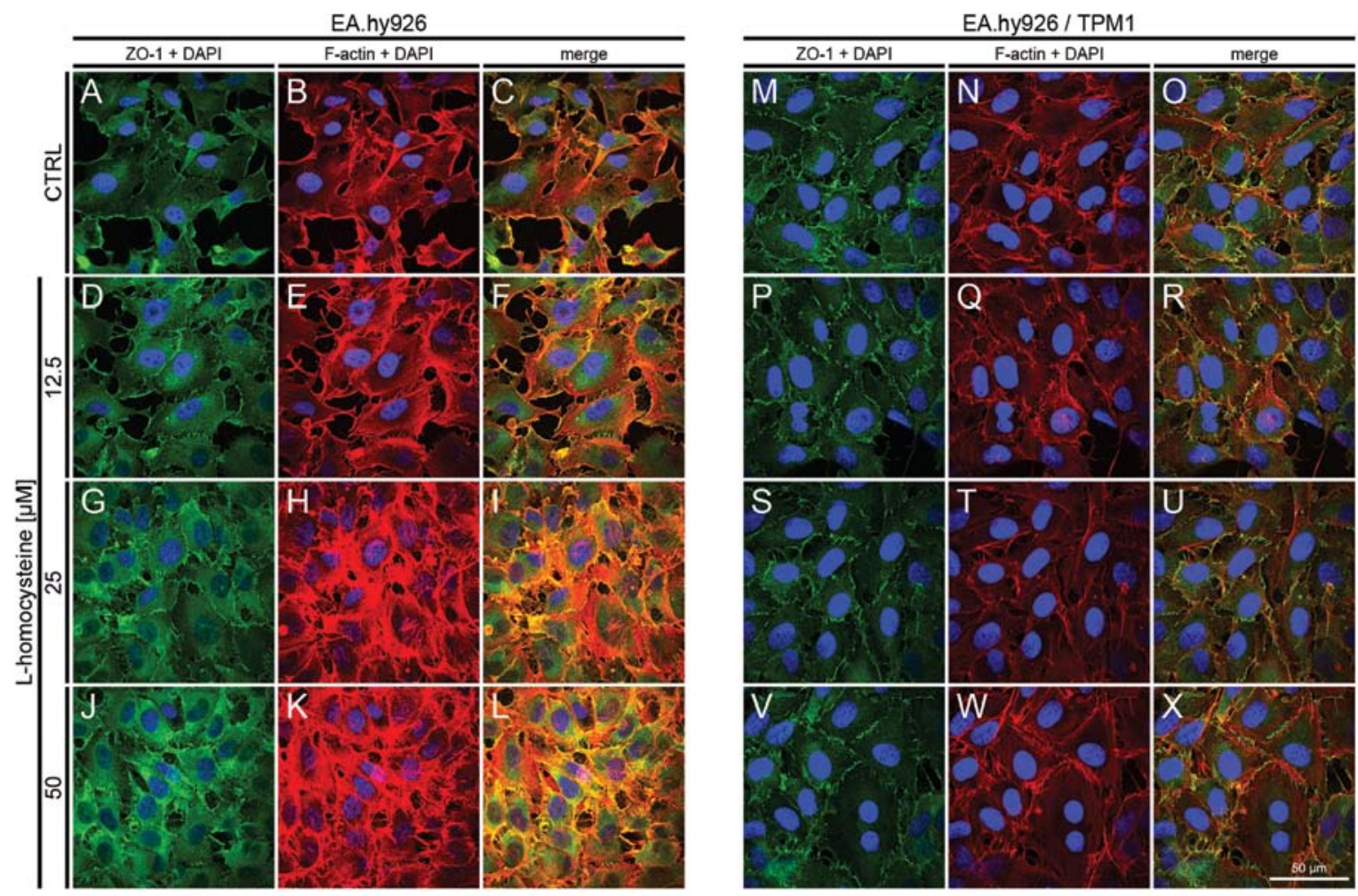

Figure 8. Effect of L-homocysteine thiolactone hydrochloride on fluorescence staining of ZO-1 and F-actin. EA.hy926 cells transfected with the empty plasmid or the plasmid with subcloned cDNA of tropomyosin-1 $(\alpha)$ isoform 1 were treated with L-homocysteine thiolactone hydrochloride for $24 \mathrm{~h}$, fixed and double-stained for the ZO-1 and F-actin. Nuclei were counterstained with DAPI. Images were captured at the focal plane of junctional ZO-1 under a confocal microscope. Bar, $50 \mu \mathrm{m}$. (A-C) Control EA.hy926 cells transfected with the empty plasmid. (D-F) EA.hy926 cells transfected with the empty plasmid and treated with $12.5 \mu \mathrm{M} \mathrm{L}$-homocysteine thiolactone hydrochloride. (G-I) EA.hy926 cells transfected with the empty plasmid and treated with $25 \mu \mathrm{M}$ L-homocysteine thiolactone hydrochloride. (J-L) EA.hy926 cells transfected with the empty plasmid and treated with $50 \mu$ M L-homocysteine thiolactone hydrochloride. (M-O) Control EA.hy926 cells transfected with the plasmid encoding tropomyosin-1 $(\alpha)$ isoform 1. (P-R) EA.hy926 cells transfected with the plasmid encoding tropomyosin-1 $(\alpha)$ isoform 1 and treated with $12.5 \mu \mathrm{M} \mathrm{L}$-homocysteine thiolactone hydrochloride. (S-U) EA.hy926 cells transfected with the plasmid encoding tropomyosin-1 $(\alpha)$ isoform 1 and treated with $25 \mu \mathrm{M} \mathrm{L}$-homocysteine thiolactone hydrochloride. (V-X) EA.hy926 cells transfected with the plasmid encoding tropomyosin-1 $(\alpha)$ isoform 1 and treated with $50 \mu \mathrm{M}$ L-homocysteine thiolactone hydrochloride. (A, D, G and J; M, P, S and V) ZO-1 + DAPI. (B, E, H and K; N, Q, T and W) F-actin + DAPI. (C, F, I and L; O, R, U and X) Merge. TPM1, tropomyosin-1.

and in those not overexpressing tropomyosin-1 was investigated using a laser scanning confocal microscope. The images were acquired in confocal mode at the focal plane of junctional ZO-1. Fluorescence double staining of ZO-1 and F-actin in the EA.hy926 cells transfected with the empty plasmid revealed an increase in the fluorescence labeling of ZO-1 following treatment with all doses of L-homocysteine in comparison to the control (Fig. 8A, D, G and J). The weakest labeling intensity was observed in the control cells, the ZO-1 fluorescence at the cell periphery area was only slightly higher than that in the central area of the cells (Fig. 8A). Similarly, F-actin labeling at the focal plane of ZO-1 revealed a dose-dependent increase in fluorescence following treatment with L-homocysteine in the form of thick stress fibers, particularly at the doses of 25 and $50 \mu \mathrm{M}$ L-homocysteine (Fig. 8B, E, H and K). Furthermore, the extracellular spaces were reduced in size following treatment with L-homocysteine in comparison to the control (Fig. 8A-L).

In the EA.hy926 cells with stabilized F-actin cytoskeleton by the overexpression of tropomyosin-1, the labeling of ZO-1 following exposure of the cells to L-homocysteine was decreased in comparison to the control cells and the cells transfected with the empty plasmid. However, ZO-1 fluorescence at the cell periphery was higher than that observed in the control cells and the cells transfected with the empty plasmid (Fig. 8M, P, S and V). Following the stabilization of F-actin, and after treatment of the cells with L-homocysteine, ZO-1 fluorescence was slightly decreased in comparison to the control, and significantly decreased, as compared to the cells transfected with the empty plasmid. Moreover, stress fibers were thinner than those observed in the control cells and the cells transfected with the empty plasmid (Fig. 8N, Q, $\mathrm{T}$ and $\mathrm{W}$ ). Additionally, the extracellular spaces were significantly reduced following F-actin stabilization, as compared to the cells transfected with the empty plasmid (Fig. 8A-X).

The measurement of the fluorescence intensity of ZO-1 and F-actin in the EA.hy926 cells and the cells overexpressing tropomyosin-1 was performed on confocal images acquired at the focal plane of ZO-1. The fluorescence intensity of F-actin and $\mathrm{ZO}-1$ was measured in whole cells and in the cell-cell interaction areas, at the focal plane of ZO-1. The analysis of the fluorescence intensity of ZO-1 measured in the whole EA.hy926 cells transfected with the empty plasmid revealed a statistically significant dose-dependent increase in comparison to the control (from 982.96 to 1194.46, $\mathrm{P}=0.0044 ; 1518.39, \mathrm{P}<0.0001$; and $1742.49, \mathrm{P}<0.0001$ for the cells treated with $12.5,25$ and $50 \mu \mathrm{M}$ L-homocysteine, respectively). Additionally, statistically significant differences were observed in the comparison between the groups (Fig. 9A). A similar increase in ZO-1 fluo- 


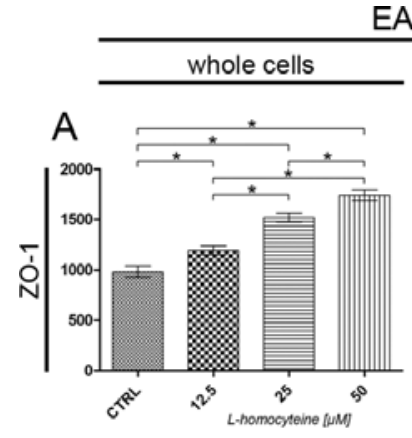

\section{EA.hy926}
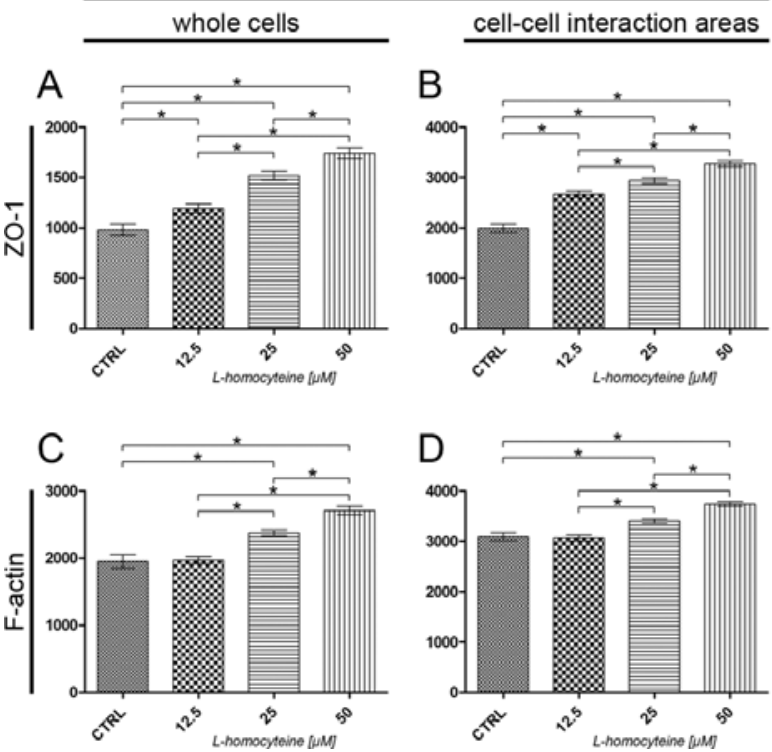

EA.hy926 / TPM1

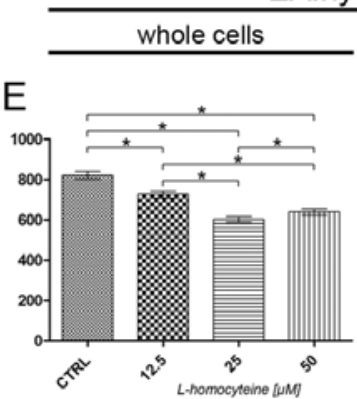
cell-cell interaction areas

$\mathrm{F}$

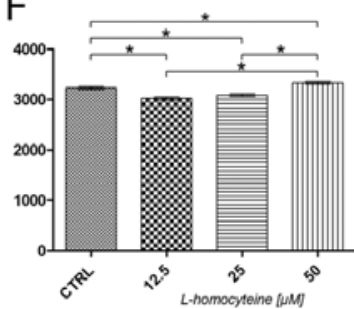

G

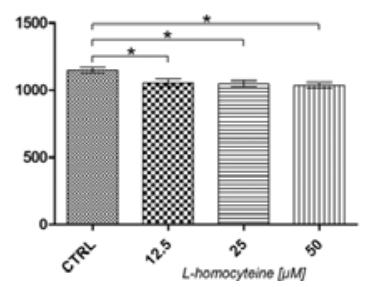

$\mathrm{H}$

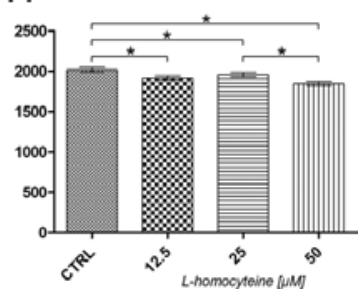

Figure 9. Effect of L-homocysteine thiolactone hydrochloride on fluorescence intensity of ZO-1 and F-actin. EA.hy926 cells transfected with the empty plasmid or the plasmid with subcloned cDNA of tropomyosin-1 $(\alpha)$ isoform 1 were treated with L-homocysteine thiolactone hydrochloride for 24 h, fixed and double-stained for the ZO-1 and F-actin. Images were captured at the focal plane of junctional ZO-1 under a confocal microscope. The analysis of fluorescence intensity of ZO-1 and F-actin was performed on confocal images in whole cells and cell-cell interaction areas. ${ }^{*}$ Statistically significant differences; $\mathrm{P}<0.05$; Mann-Whitney U test. (A and B) Fluorescence intensity of ZO-1 measured in EA.hy926 cells transfected with the empty plasmid. (C and D) Fluorescence intensity of F-actin measured in EA.hy926 cells transfected with the empty plasmid. (E and F) Fluorescence intensity of ZO-1 measured in EA.hy926 cells transfected with the plasmid encoding tropomyosin-1 $(\alpha)$ isoform 1. (G and H) Fluorescence intensity of F-actin measured in EA.hy 926 cells transfected with the plasmid encoding tropomyosin-1 ( $\alpha$ ) isoform 1. (A and C; E and G) Fluorescence intensity measured in whole cells. (B and D; F and H) Fluorescence intensity measured in cell-cell interaction areas. TPM1, tropomyosin-1; CTRL, control.

rescence in comparison to the control and a correlation between the doses of L-homocysteine were observed following the measurement of its intensity in the cell-cell interaction areas, although the values were 1.88- to 2.23-fold higher, as compared to the fluorescence intensity measured in the whole cells (Fig. 9B). A statistically significant increase in the fluorescence intensity of F-actin was observed in the whole cells following the treatment of the cells with 25 and $50 \mu \mathrm{M} \mathrm{L}$-homocysteine in comparison to the control (from 1950.04 to 2377.88, $\mathrm{P}=0.0003$; and 2708.54, $\mathrm{P}<0.0001$, respectively). Moreover, the differences between the doses of L-homocysteine were statistically significant (Fig. 9C). A similar correlation was observed in F-actin intensity measured in the cell-cell interaction areas; however, the fluorescence intensity was 1.38 - to 1.58 -fold higher than the intensity measured in the whole cells (Fig. 9D).

An analysis of the fluorescence intensity of ZO-1 measured in whole EA.hy926 cells overexpressing tropomyosin-1 revealed a statistically significant decrease following treatment of the cells with L-homocysteine (from 820.85 to 725.23 , $\mathrm{P}<0.0001 ; 602.80, \mathrm{P}<0.0001$; and 638.74, $\mathrm{P}<0.0001$ for the cells treated with 12.5, 25 and $50 \mu \mathrm{M}$ L-homocysteine, respectively). Moreover, statistically significant differences were observed between the doses of L-homocysteine. Additionally, the fluorescence intensity values were 1.65 - to 2.73 -fold lower than those in the cells transfected with the empty plasmid (Fig. 9E). There was a statistically significant decrease in the fluorescence intensity of ZO-1 measured in the cell-cell interaction areas following treatment of the EA.hy926 cells with stabilized F-actin cytoskeleton by tropomyosin-1 with 12.5 and $25 \mu \mathrm{M}$ L-homocysteine (from 3230.88 to 3020.65 , $\mathrm{P}<0.0001$; and $3083.39, \mathrm{P}=0.0023$, respectively) and an increase following treatment of the cells with $50 \mu \mathrm{M}$ L-homocysteine (to 3329.29, $\mathrm{P}=0.0170$ ), as compared to the control (Fig. 9F). Furthermore, the cell-cell interaction areas exhibited a slightly higher (1.02to 1.62-fold) fluorescence intensity of $\mathrm{ZO}-1$ than that observed in the cells without stabilized F-actin (Fig. 9F).

The measurement of F-actin fluorescence intensity at the focal plane of ZO-1 in the whole cells overexpressing tropomyosin-1 revealed a statistically significant decrease, as compared to the control (from 1148.77 to $1056.86, \mathrm{P}=0.0026$; 1048.67, $\mathrm{P}=0.0021$; and $1036.54, \mathrm{P}=0.0012$ for the cells treated with $12.5,25$ and $50 \mu \mathrm{M}$ L-homocysteine, respectively). The differences between the results obtained from the cells treated with L-homocysteine were statistically insignificant (Fig. 9G). A similar correlation was observed in F-actin intensity measured in the cell-cell interaction areas, although the fluorescence intensity was 1.76 - to 1.87 -fold higher than the intensity measured in the whole cells (Fig. 9H).

Analysis of relative fluorescence changes of junctional $\alpha$-catenin, $\beta$-catenin and $Z O-1$. The effect of L-homocysteine on relative fluorescence changes of $\alpha$-catenin, $\beta$-catenin and ZO-1 in the EA.hy926 cells overexpressing tropomyosin-1 and in those not overexpressing tropomyosin- 1 was investigated by using a laser scanning confocal microscope. The images were acquired in confocal mode at the focal plane of the junctional proteins. The relative fluorescence values of the junctional proteins were calculated by dividing the fluorescence intensity values measured in the cell-cell interaction areas by the median of the fluorescence intensity measured in the cell-cell interaction areas of the control and the transfected cells or the cells transfected with the empty plasmid. 

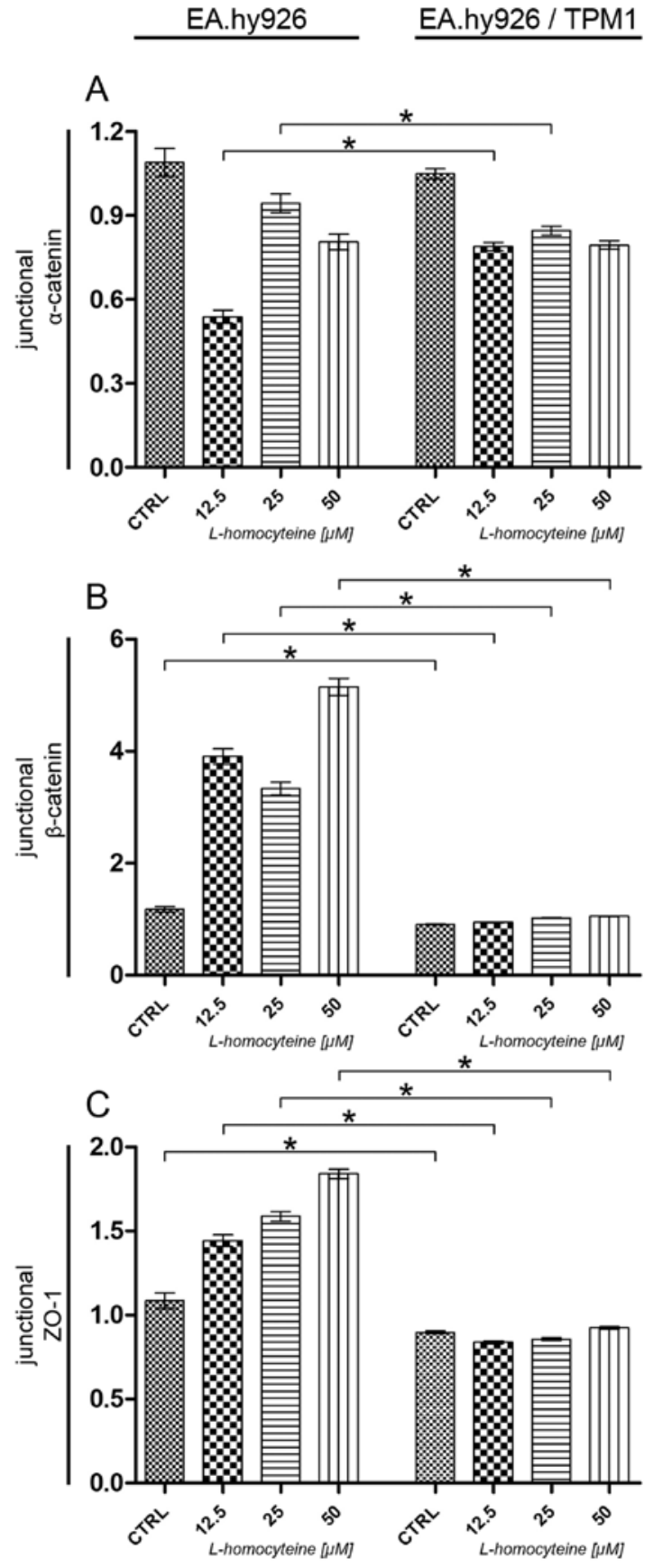

Figure 10. Effect of L-homocysteine thiolactone hydrochloride on relative fluorescence of junctional $\alpha$-catenin, $\beta$-catenin and ZO-1. EA.hy926 cells transfected with the empty plasmid or the plasmid with subcloned cDNA of tropomyosin-1 $(\alpha)$ isoform 1 were treated with L-homocysteine thiolactone hydrochloride for $24 \mathrm{~h}$, fixed and stained for the $\alpha$-catenin, $\beta$-catenin or ZO-1. Images were captured at the focal plane of junctional proteins under a confocal microscope. The analysis of fluorescence intensity junctional $\alpha$-catenin, $\beta$-catenin or ZO-1 was performed on confocal images in cell-cell interaction areas. The relative fluorescence values of junctional proteins were calculated by dividing the fluorescence intensity values measured in the cell-cell interaction areas by the median of fluorescence intensity measured in the cell-cell interaction areas of the control cells and the cells transfected with the empty plasmid or the plasmid encoding tropomyosin-1 $(\alpha)$ isoform 1. *Statistically significant differences between cells transfected with the empty plasmid or the plasmid encoding tropomyosin-1 $(\alpha)$ isoform 1 ; P $<0.05$; Mann-Whitney U test (A) Relative fluorescence of junctional $\alpha$-catenin measured in EA.hy926 cells transfected with empty plasmid or plasmid encoding tropomyosin-1 $(\alpha)$ isoform 1. (B) Relative fluorescence of junctional $\beta$-catenin measured in EA.hy926 cells transfected with the empty plasmid or the plasmid encoding tropomyosin-1 $(\alpha)$ isoform 1. (C) Relative fluorescence of junctional ZO-1 measured in EA.hy926 cells transfected with the empty plasmid or plasmid encoding tropomyosin-1 $(\alpha)$ isoform 1. TPM1, tropomyosin-1; CTRL, control.
An analysis of junctional $\alpha$-catenin relative fluorescence revealed a statistically significant increase following treatment of the cells overexpressing tropomyosin- 1 with $12.5 \mu \mathrm{M}$ L-homocysteine in comparison to the cells transfected with the empty plasmid treated with the same L-homocysteine dose (0.54-0.79, P<0.0001). Moreover, a comparison of both types of cells following treatment with $25 \mu \mathrm{M} \mathrm{L}$-homocysteine revealed a statistically significant decrease in the relative fluorescence of junctional $\alpha$-catenin $(0.94-0.84, \mathrm{P}=0.0036)$. Furthermore, no statistically significant differences were observed between the control cells and the cells treated with $50 \mu \mathrm{M}$ L-homocysteine (Fig. 10A).

As regards junctional $\beta$-catenin, analysis revealed a statistically significant decrease in the relative fluorescence of $\beta$-catenin in the transfected control cells and the cells treated with all L-homocysteine doses, as compared to the cells transfected with the empty plasmid $(1.17-0.90, \mathrm{P}=0.0031 ; 3.90-0.94$, $\mathrm{P}<0.0001 ; 3.33-1.02, \mathrm{P}<0.0001$; and 5.15-1.05, $\mathrm{P}<0.0001$ for the control cells, and the cells treated with $12.5,25$ and $50 \mu \mathrm{M}$ L-homocysteine, respectively) (Fig. 10B).

Similar to the results obtained for junctional $\beta$-catenin, analysis revealed a statistically significant decrease in the relative fluorescence of ZO-1 in the control cells overexpressing tropomyosin-1 and the same cells treated with all L-homocysteine doses, as compared to the cells transfected with the empty plasmid (1.08-0.90, $\mathrm{P}=0.0071$; and 1.44-0.84, $\mathrm{P}<0.0001$; $1.59-0.85, \mathrm{P}<0.0001$; or $1.84-0.92, \mathrm{P}<0.0001$ for the control cells, and the cells treated with $12.5,25$ and $50 \mu \mathrm{M}$ L-homocysteine, respectively) (Fig. 10C).

\section{Discussion}

Atherosclerosis affects the arteries and results in heart disease and myocardial infarction. Atherosclerosis results from injury to the artery endothelium caused by mechanical and environmental factors, and the resulting inflammatory response in the vessel wall (6). Previous clinical and experimental studies have indicated that mild to moderate increases in the plasma homocysteine concentration are casual risk factors for vascular disease (7-10). As it has been previously shown by Plazar and Jurdana, the normal and abnormal homocysteine levels are set by individual laboratories. However, the concentration of homocysteine is considered normal at levels $<13 \mu \mathrm{M}$, levels between 13 and $60 \mu \mathrm{M}$ are considered moderately elevated, and levels $>60$ to $100 \mu \mathrm{M}$ are considered significantly elevated (37). Moreover, the total plasma homocysteine concentrations during hyperhomocysteinemia are between 12 and $30 \mu \mathrm{M}$, with gender differences being present. Higher values are measured in males, while the presence of estrogen in females determines the plasma concentration and following menopause, the blood levels of homocysteine are similar to those observed in males (38). A number of in vitro studies have indicated that at micromolar concentrations, homocysteine exerts anti-angiogenic effects and decreases the bioavailability of endothelium-derived nitric oxide $(39,40)$. At higher micromolar concentrations, homocysteine has been shown to induce apoptosis and decrease the migration of endothelial cells (40-43).

In this study, we used $12.5,25$ and $50 \mu \mathrm{ML}$-homocysteine thiolactone hydrochloride to investigate the effect L-homocysteine 
on endothelial EA.hy926 cells in the context of changes in cell-cell junctions. Moreover, through the overexpression of tropomyosin-1, we stabilized F-actin cytoskeleton to determine whether organizational changes of F-actin play a role in endothelial injury. Mercié et al showed that homocysteine thiolactone in the range of 50-200 $\mu \mathrm{M}$ induced endothelial cell apoptosis in a concentration-dependent manner, independently of the caspase pathway. Additionally, the apoptosis was not influenced by the serum concentration in the culture medium, and inhibitors such as leupeptin, fumosinin B1, catalase, or z-VAD-fmk were able to prevent homocysteine-induced apoptic cell death (44). On the contrary, in the present study, no statistically significant differences were observed between the percentage of live and early apoptotic cells following treatment with L-homocysteine as compared to the control. In the study by Rodríguez-Nieto et al, it was shown that $5 \mathrm{mM}$ homocysteine had only a weak cyotoxic effect on BAE cells, as revealed both by cell counting and by negative results obtained by TUNEL assay and caspase-3 activity assay (45). Additionally, our results revealed that the stabilization of F-actin cytoskeleton caused a significant decrease in the percentage of late apoptotic and necrotic cells following treatment with L-homocysteine as compared to the cells transfected with the empty plasmid. Furthermore, the percentage of early apoptotic cells was decreased following treatment of the cells with $25 \mu \mathrm{M}$ L-homocysteine in comparison to the EA.hy926 cells not overexpressing tropomyosin-1, treated with the same dose of L-homocysteine. However, no statistically significant differences were observed between the percentage of live cells as compared to the cells overexpressing tropomyosin- 1 and those not overexpressing tropomyosin-1. Our results demonstrate that through the stabilization of F-actin, tropomyosin-1 protects EA.hy926 cells against late apoptosis and necrosis.

As regards endothelial cell mobility following treatment with homocysteine, homocysteine has been shown to inhibit cell migration and invasion (45). Our study demonstrated an increase in the cell mobility of EA.hy926 cells overexpressing tropomyosin-1, as compared to the EA.hy926 cells transfected with the empty plasmid after $12 \mathrm{~h}$ from the time when the wound was formed. This suggests that the stabilization of F-actin does not affect the migratory potential of cells, and consequently protects the EA.hy926 cells against the L-homocysteine-induced decrease in cell mobility. Similar results were obtained by Wejksza et al following treatment of bovine aorta endothelial cell cultures with kynurenic acid (KYNA). The above cited authors showed that KYNA exerted a protective effect against the homocysteine-induced impairment of endothelial cells. The addition of $0.01 \mathrm{mM} \mathrm{KYNA}$ to the cell culture containing $0.5 \mathrm{mM}$ DL-homocysteine significantly increased endothelial cell migration and proliferation, which were diminished by homocysteine. KYNA also protected the cells against homocysteine-induced cytotoxicity (46).

The filamentous actin protein includes a variety of different structures that are crucial in many aspects of cell physiology, including the maintenance of cell shape and polarity, which are important in the formation of cellular junctions (4). As has previously been shown by Zhang, the cooperation of junctional actin and F-actin thin bundles lead to the overall morphological changes and develop a polarized epithelial cell shape (5). Similar to epithelial cells, endothelial cells have specialized junctional regions that are comparable to adherens junctions and tight junctions. However, in the endothelium, tight junctions are frequently intermixed with adherens junctions along the intercellular cleft (13). The actin cytoskeleton and associated proteins play a vital role in cell-cell adhesion (14). As it has been shown in a number of studies, there is the possibility of interaction between the cytoplasmic tails of junctional proteins and both cytoskeletal and signaling proteins, which allows the anchorage of the adhesion proteins to F-actin and the transfer of intracellular signals inside the cell (15-18). The association with actin is required not only for the stabilization of junctions, but also for the dynamic regulation of junction opening and closure. Although a key role of the actin cytoskeleton in the formation and maintenance of adherens junctions has been recognized, particularly as regards the molecular linkages between cadherins and actin filaments, the structural organization and specific roles of the actin cytoskeleton at adherens junctions remain unknown, particularly in endothelial cells (14,19-21).

In endothelial adherens junctions, the transmembrane vascular endothelial (VE)-cadherin binds the cytoplasmic proteins, $\beta$-catenin and p120-catenin. $\beta$-catenin also binds $\alpha$-catenin, which could link the adheren junction complex to actin filaments (47). However, the established model of a direct link between cortical actin filaments (F-actin) and $\alpha$-catenin in adherens junctions in epithelial cells has been questioned. It has been suggested that the binding of $\alpha$-catenin to $\beta$-catenin and to F-actin is mutually exclusive, and that the role of $\alpha$-catenin is the stabilization of adherens junctions by regulating actin polymerization as opposed to linking F-actin to adherens junctions $(48,49)$. Our study demonstrated that the stabilization of F-actin by the overexpression of tropomyosin-1 in EA.hy926 cells significantly increased the expression of junctional $\beta$-catenin, measured by fluorescence intensity changes, compared to the cells not overexpressing tropomyosin-1. Similarly, the fluorescence intensity of junctional $\alpha$-catenin was also increased in the cells with stabilized F-actin cytoskeleton. However, this increase was only slightly higher than that observed in the EA.hy926 cells not overexpressing tropomyosin-1. Moreover, following an analysis of the increase of $\alpha$-catenin fluorescence by relative fluorescence intensity analysis, the results revealed a similar distribution following treatment with L-homocysteine in the EA.hy926 cells with and without stabilized F-actin cytoskeleton. Together with the decreased fluorescence intensity of junctional F-actin measured in the cell-cell contact area at the focal plane of $\alpha$-catenin, it can be hypothesized that $\alpha$-catenin may participate in the suppression of actin polymerization in the area of cell-cell junctions. Our results are in agreement with those presented in the study by Drees et al, that $\alpha$-catenin can locally regulate actin dynamics and organization. Furthermore, we confirm the hypothesis that $\alpha$-catenin directly regulates actin filament organization by suppressing Arp2/3-mediated actin polymerization, possibly by competing with the Arp $2 / 3$ complex for binding to actin filaments (48). However, Millán et al suggested that endothelial discontinuous adheren junctions formed by complexes of VE-cadherin, $\alpha$-catenin, $\beta$-catenin and p120-catenin, can be physically linked to actin stress fibers (50). However, in this study, the analysis of $\alpha$-catenin and $\beta$-catenin relative fluorescence in the cells with stabilized F-actin cytoskeleton, confirm the hypotheses made by Drees et al and Yamada et al, that $\alpha$-catenin does not bind simultaneously to the E-cadherin- $\beta$-catenin complex and actin filaments $(48,49)$. 
ZO-1 is a member of the membrane-associated guanylate kinase homolog (MAGUK) family of membrane-associated signaling molecules that binds directly to both cytosolic and transmembrane components of the tight junction and is believed to organize these proteins within the apical junctional complex (51). It also binds directly to F-actin (52-54). Although the functional relevance of this interaction is unknown, it is believed that ZO-1 limits solute permeability in established tight junctions, possibly by forming a stabilizing link between the barrier and perijunctional actomyosin ring (55). Van Itallie et al also indicated that F-actin localization is significantly altered in ZO-1 knockdown cells, with an increase in actin staining at the apical junctional complex and into scattered apical dots (55). The analysis of relative fluorescence presented in this study revealed a statistically significant decrease in the relative fluorescence of ZO-1 in the cell-cell junction area among the cells with stabilized F-actin cytoskeleton in comparison to the cells not overexpressing tropomyosin-1. Our results suggest that ZO-1, similar to $\beta$-catenin, can bind to F-actin directly, and stabilize the endothelial barrier function through the stabilization of F-actin. However, as has previously been suggested by Van Itallie et al (55), ZO-1 has very specific effects on actin dynamics at the apical junctional complex, possibly by localizing the activity of cytoskeletal proteins, such as $\alpha$-actinin, $\alpha$-catenin or shroom 2 (52,55-57). Furthermore, we observed that through the stabilization of F-actin, the localization of junctional ZO-1 did not change drastically following treatment with L-homocysteine, as was confirmed by relative fluorescence analysis; these results confirm those presented in the study by Fanning et al (51).

In conclusion, the present study demonstrates that the stabilization of F-actin does not affect the migratory potential of cells, and consequently protects the EA.hy926 cells against the L-homocysteine-induced decrease in cell mobility. Moreover, the data presented in this study suggest that $\alpha$-catenin participates in the suppression of actin polymerization in the area of cell-cell junctions. Our data are in agreement with those presented in the studies by Drees et al and Yamada et al, namely that $\alpha$-catenin does not bind simultaneously to the E-cadherin- $\beta$-catenin complex and actin filaments $(48,49)$. Furthermore, we hypothesize that the stabilization of F-actin strengthens adherens junctions and tight junctions by increasing the number of cell-cell junctions due to the amplification of $\beta$-catenin and the $\mathrm{ZO}-1$ fluorescence signal. However, ZO-1 stabilizes the endothelial barrier function through the stabilization of F-actin and F-actin itself stabilizes the localization of ZO-1.

\section{Acknowledgements}

This study was supported by the Polish National Science Center (NCN) under the Grant no. N N401 596140.

\section{References}

1. Pollard TD: Cytoskeletal functions of cytoplasmic contractile proteins. J Supramol Struct 5: 317-334, 1976.

2. Clarke M and Spudich JA: Nonmuscle contractile proteins: the role of actin and myosin in cell motility and shape determination. Annu Rev Biochem 46: 797-822, 1977.

3. Stossel TP: Contractile proteins in cell structure and function. Annu Rev Med 29: 427-457, 1978.
4. Stricker J, Falzone T and Gardel ML: Mechanics of the F-actin cytoskeleton. J Biomech 43: 9-14, 2010.

5. Zhang J, Betson M, Erasmus J, Zeikos K, Bailly M, Cramer LP and Braga VM: Actin at cell-cell junctions is composed of two dynamic and functional populations. J Cell Sci 118: 5549-5562, 2005.

6. Homeister JW and Willis MS: Atherosclerosis: Pathogenesis, Genetics and Experimental Models. In: Encyclopedia of Life Sciences 2010. John Wiley \& Sons Ltd., Chichester, 2010.

7. Boushey CJ, Beresford SA, Omenn GS and Motulsky AG: A quantitative assessment of plasma homocysteine as a risk factor for vascular disease. Probable benefits of increasing folic acid intakes. JAMA 274: 1049-1057, 1995.

8. Nygård O, Vollset SE, Refsum H et al: Total plasma homocysteine and cardiovascular risk profile. The Hordaland Homocysteine Study. JAMA 274: 1526-1533, 1995.

9. Ueland PM, Refsum H, Beresford SA and Vollset SE: The controversy over homocysteine and cardiovascular risk. Am J Clin Nutr 72: 324-332, 2000.

10. Tehlivets O: Homocysteine as a risk factor for atherosclerosis: is its conversion to s-adenosyl-L-homocysteine the key to deregulated lipid metabolism? J Lipids 2011: 702853, 2011.

11. McDowell IF and Lang D: Homocysteine and endothelial dysfunction: a link with cardiovascular disease. J Nutr 130: 369S-372S, 2000.

12. Heydrick SJ, Weiss N, Thomas SR, Cap AP, Pimentel DR, Loscalzo J and Keaney JF Jr: L-Homocysteine and L-homocystine stereospecifically induce endothelial nitric oxide synthasedependent lipid peroxidation in endothelial cells. Free Radic Biol Med 36: 632-640, 2004.

13. Dejana E: Endothelial cell-cell junctions: happy together. Nat Rev Mol Cell Biol 5: 261-270, 2004.

14. Hoelzle MK and Svitkina T: The cytoskeletal mechanisms of cell-cell junction formation in endothelial cells. Mol Biol Cell 23: 310-323, 2012.

15. Bazzoni G, Martínez Estrada O and Dejana E: Molecular structure and functional role of vascular tight junctions. Trends Cardiovasc Med 9: 147-152, 1999.

16. Braga VM: Cell-cell adhesion and signalling. Curr Opin Cell Biol 14: 546-556, 2002.

17. Matter $\mathrm{K}$ and Balda MS: Signalling to and from tight junctions. Nat Rev Mol Cell Biol 4: 225-236, 2003.

18. Wheelock MJ and Johnson KR: Cadherin-mediated cellular signaling. Curr Opin Cell Biol 15: 509-514, 2003.

19. Harris ES and Nelson WJ: VE-cadherin: at the front, center, and sides of endothelial cell organization and function. Curr Opin Cell Biol 22: 651-658, 2010.

20. Harris TJ and Tepass U: Adherens junctions: from molecules to morphogenesis. Nat Rev Mol Cell Biol 11: 502-514, 2010.

21. Yonemura S: Cadherin-actin interactions at adherens junctions. Curr Opin Cell Biol 23: 515-522, 2011.

22. Nwariaku FE, Liu Z, Zhu X et al: NADPH oxidase mediates vascular endothelial cadherin phosphorylation and endothelial dysfunction. Blood 104: 3214-3220, 2004.

23. Kinumi T, Ogawa Y, Kimata J, Saito Y, Yoshida Y and Niki E: Proteomic characterization of oxidative dysfunction in human umbilical vein endothelial cells (HUVEC) induced by exposure to oxidized LDL. Free Radic Res 39: 1335-1344, 2005.

24. Xu Y, Arai H, Murayama T, Kita T and Yokode M: Hypercholesterolemia contributes to the development of atherosclerosis and vascular remodeling by recruiting bone marrow-derived cells in cuff-induced vascular injury. Biochem Biophys Res Commun 363: 782-787, 2007.

25. Javanmard SH and Dana N: The effect of interferon $\gamma$ on endothelial cell nitric oxide production and apoptosis. Adv Biomed Res 1: 69, 2012.

26. Bouïs D, Hospers GA, Meijer C, Molema G and Mulder NH: Endothelium in vitro: a review of human vascular endothelial cell lines for blood vessel-related research. Angiogenesis 4: 91-102, 2001.

27. Edgell CJ, Reisner HM and Graham JB: Endothelial cell hybrids and the suspension of factor VIII related antigen expression. Br J Haematol 46: 613-620, 1980.

28. Edgell CJ, Haizlip JE, Bagnell CR, Packenham JP, Harrison P, Wilbourn B and Madden VJ: Endothelium specific Weibel-Palade bodies in a continuous human cell line, EA.hy926. In Vitro Cell Dev Biol 26: 1167-1172, 1990.

29. Claise C, Chalas J, Edeas M, Abella A, Khalfoun Y, Laurent D and Lindenbaum A: Comparison of oxidized low-density lipoprotein toxicity on EA.hy 926 cells and human vein endothelial cells: influence of antioxidant systems. Cell Mol Life Sci 53: 156-161, 1997. 
30. Vrekoussis T, Stathopoulos EN, De Giorgi U et al: Modulation of vascular endothelium by imatinib: a study on the EA.hy 926 endothelial cell line. J Chemother 18: 56-65, 2006.

31. Rosenkranz AC, Lob H, Breitenbach T, Berkels R and Roesen R Endothelial antioxidant actions of dihydropyridines and angiotensin converting enzyme inhibitors. Eur J Pharmacol 529: 55-62, 2006.

32. Pittenger MF, Kazzaz JA and Helfman DM: Functional properties of non-muscle tropomyosin isoforms. Curr Opin Cell Biol 6 : 96-104, 1994.

33. Perry SV: Vertebrate tropomyosin: distribution, properties and function. J Muscle Res Cell Motil 22: 5-49, 2001.

34. Cooper JA. Actin dynamics: tropomyosin provides stability. Curr Biol 12: R523-R525, 2002.

35. Gunning PW, Schevzov G, Kee AJ and Hardeman EC: Tropomyosin isoforms: divining rods for actin cytoskeleton function. Trends Cell Biol 15: 333-341, 2005.

36. Lin JJ, Eppinga RD, Warren KS and McCrae KR: Human tropomyosin isoforms in the regulation of cytoskeleton functions. Adv Exp Med Biol 644: 201-222, 2008.

37. Plazar $\mathrm{N}$ and Jurdana M: Hyperhomocysteinemia: relation to cardiovascular disease and venous thromboembolism. In: Pathophysiology and Clinical Aspects of Venous Thromboembolism in Neonates, Renal Disease and Cancer Patients. Abdelaal MA (ed.). InTech, Rijeka, pp17-34, 2012.

38. Ridker PM, Manson JE, Buring JE, Shih J, Matias M and Hennekens CH: Homocysteine and risk of cardiovascular disease among postmenopausal women. JAMA 281: 1817-1821, 1999.

39. Upchurch GR Jr, Welch GN, Fabian AJ, Freedman JE, Johnson JL, Keaney JF Jr and Loscalzo J: Homocyst(e)ine decreases bioavailable nitric oxide by a mechanism involving glutathione peroxidase. J Biol Chem 272: 17012-17017, 1997.

40. Nagai Y, Tasaki H, Takatsu H, Nihei S, Yamashita K, Toyokawa T and Nakashima Y: Homocysteine inhibits angiogenesis in vitro and in vivo. Biochem Biophys Res Commun 281: 726-731, 2001.

41. Zhang C, Cai Y, Adachi MT, Oshiro S, Aso T, Kaufman RJ and Kitajima S: Homocysteine induces programmed cell death in human vascular endothelial cells through activation of the unfolded protein response. J Biol Chem 276: 35867-35874, 2001.

42. Lee SJ, Kim KM, Namkoong S et al: Nitric oxide inhibition of homocysteine-induced human endothelial cell apoptosis by down-regulation of p53-dependent Noxa expression through the formation of S-nitrosohomocysteine. J Biol Chem 280: 5781-5788, 2005

43. Suhara T, Fukuo K, Yasuda O et al: Homocysteine enhances endothelial apoptosis via upregulation of Fas-mediated pathways. Hypertension 43: 1208-1213, 2004.
44. Mercié P, Garnier O, Lascoste L et al: Homocysteine-thiolactone induces caspase-independent vascular endothelial cell death with apoptotic features. Apoptosis 5: 403-411, 2000.

45. Rodríguez-Nieto S, Chavarría T, Martínez-Poveda B, Sánchez-Jiménez F, Rodríguez Quesada A and Medina MA: Anti-angiogenic effects of homocysteine on cultured endothelial cells. Biochem Biophys Res Commun 293: 497-500, 2002.

46. Wejksza K, Rzeski W and Turski WA: Kynurenic acid protects against the homocysteine-induced impairment of endothelial cells. Pharmacol Rep 61: 751-756, 2009.

47. Vincent PA, Xiao K, Buckley KM and Kowalczyk AP: VE-cadherin: adhesion at arm's length. Am J Physiol Cell Physiol 286: C987-C997, 2004.

48. Drees F, Pokutta S, Yamada S, Nelson WJ and Weis WI: $\alpha$-catenin is a molecular switch that binds E-cadherin- $\beta$-catenin and regulates actin-filament assembly. Cell 123: 903-915, 2005.

49. Yamada S, Pokutta S, Drees F, Weis WI and Nelson WJ: Deconstructing the cadherin-catenin-actin complex. Cell 123: 889-901, 2005

50. Millán J, Cain RJ, Reglero-Real N et al: Adherens junctions connect stress fibres between adjacent endothelial cells. BMC Biol 8: 11, 2010.

51. Fanning AS, Ma TY and Anderson JM: Isolation and functional characterization of the actin binding region in the tight junction protein ZO-1. FASEB 16: 1835-1837, 2002.

52. Itoh M, Nagafuchi A, Moroi S and Tsukita S: Involvement of ZO-1 in cadherin-based cell adhesion through its direct binding to alpha catenin and actin filaments. J Cell Biol 138: 181-192, 1997.

53. Fanning AS, Jameson BJ, Jesaitis LA and Anderson JM: The tight junction protein $\mathrm{ZO}-1$ establishes a link between the transmembrane protein occludin and the actin cytoskeleton J Biol Chem 273: 29745-29753, 1998.

54. Wittchen ES, Haskins J and Stevenson BR: Protein interactions at the tight junction. Actin has multiple binding partners, and ZO-1 forms independent complexes with ZO-2 and ZO-3. J Biol Chem 274: 35179-35185, 1999.

55. Van Itallie CM, Fanning AS, Bridges A and Anderson JM: ZO-1 stabilizes the tight junction solute barrier through coupling to the perijunctional cytoskeleton. Mol Biol Cell 20: 3930-3940, 2009.

56. Chen VC, Li X, Perreault H and Nagy JI: Interaction of zonula occludens-1 (ZO-1) with alpha-actinin-4: application of functional proteomics for identification of PDZ domain-associated proteins. J Proteome Res 5: 2123-2134, 2006.

57. Etournay R, Zwaenepoel I, Perfettini I, Legrain P, Petit $C$ and El-Amraoui A: Shroom2, a myosin-VIIa- and actin-binding protein, directly interacts with ZO-1 at tight junctions J Cell Sci 120: 2838-2850, 2007. 